Document downloaded from:

http://hdl.handle.net/10251/94084

This paper must be cited as:

García Trenco, A.; Martinez Feliu, A. (2012). Direct synthesis of DME from syngas on hybrid CuZnAl/ZSM-5 catalysts: New insights into the role of zeolite acidity. Applied Catalysis A General. 411:170-179. doi:10.1016/j.apcata.2011.10.036

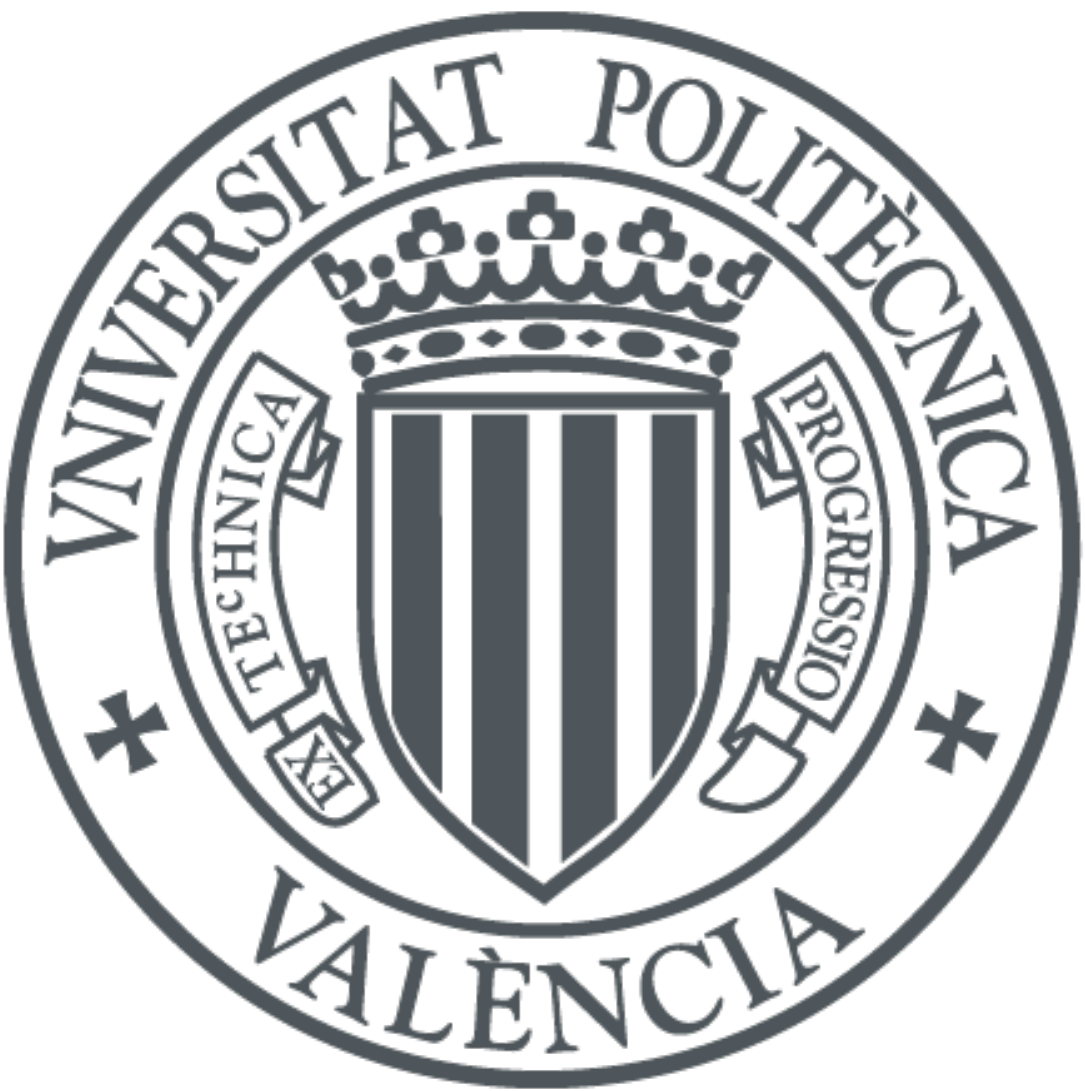

The final publication is available at

https://doi.org/10.1016/j.apcata.2011.10.036

Copyright Elsevier

Additional Information 


\title{
Direct synthesis of DME from syngas on hybrid CuZnAl/ZSM-5 catalysts: new insights into the role of zeolite acidity
}

\author{
Andrés García-Trenco, Agustín Martínez*
}

Instituto de Tecnología Química, UPV-CSIC, Avda. de los Naranjos s/n, 46022, Valencia, Spain.

*Corresponding author: amart@itq.upv.es (A. Martínez). 


\section{Abstract}

A commercial HZSM-5 zeolite $(\mathrm{Si} / \mathrm{Al}=16)$ was submitted to mild acid treatment and partial exchange of $\mathrm{H}^{+}$by $\mathrm{Na}^{+}$and $\mathrm{Co}^{2+}$ cations in order to produce samples with different amounts and strengths of Brønsted and Lewis acid sites. The acid properties of the zeolites were determined by both FTIR of adsorbed pyridine and $\mathrm{NH}_{3}-\mathrm{TPD}$. Then, hybrid catalysts comprising a $\mathrm{CuO} / \mathrm{ZnO} / \mathrm{Al}_{2} \mathrm{O}_{3}(\mathrm{CZA})$ methanol synthesis catalyst and the original and treated zeolites were prepared in order to analyze the impact of the zeolite acidity in the efficiency of the hybrids for the syngas-to-DME reaction (so-called STD process). Independent methanol dehydration experiments on the bare zeolites and STD runs under methanol dehydration-controlled conditions were performed using hybrids with low zeolite concentration (CZA:zeolite mass ratio of 10:1) prepared by mixing the pre-pelletized CZA and zeolite components. The results revealed that both strong Brønsted and EFAL-related strong Lewis acid sites are the likely zeolite active sites for methanol dehydration at the typical STD temperature of $260^{\circ} \mathrm{C}$. Interestingly, we have found that different conclusions regarding the effect of zeolite acidity may be reached depending on the specific method used for preparing the hybrid catalysts when the overall STD process becomes controlled by the methanol synthesis rate on the $\mathrm{Cu}$ based catalyst (i.e. using hybrids with a CZA:zeolite mass ratio of 2:1, that is, with an "excess" of acid sites). Thus, for hybrids prepared by mixing the pre-pelletized components, the same CO conversion and product selectivity (with values approaching those predicted by the thermodynamic equilibrium at the studied conditions) with no signs of deactivation during at least $50 \mathrm{~h}$ on stream was attained irrespective of the zeolite acidity. By contrast, significant differences in catalyst stability were found for hybrids prepared by grinding the component powders prior to pelletizing, pointing towards the occurrence of detrimental interactions between the CZA and zeolite components during the grinding preparation stage. Moreover, the different TOS behaviors observed between these hybrids depending on the particular zeolite used suggested that the kind and extent of such detrimental interactions should be tightly related to the acidity and/or chemical composition of the ZSM-5 zeolite.

Keywords: Synthesis gas; syngas-to-dimethyl ether; STD process; CuZnAl catalyst; ZSM-5; zeolite acidity. 


\section{INTRODUCTION}

Dimethyl ether (DME) is a well-known building block in the production of valuable chemicals such as methyl acetate and dimethyl sulfate, as well as of petrochemicals (BTX aromatics and light olefins) and conventional fuels by replacing methanol as raw material. Moreover, DME is attracting increasing interest as a potential eco-friendly substitute for petroleum-derived diesel fuel given its high cetane number (55-60), low auto-ignition temperature, and reduced emissions of hazardous compounds such as $\mathrm{NO}_{\mathrm{x}}, \mathrm{SO}_{\mathrm{x}}$, and particulate matter [1,2]. DME has also great potential as energy carrier, for instance, as a residential fuel for heating and cooking replacing LPG (liquefied petroleum gas), as fuel for power generation using DME-fired turbines, or as a source of hydrogen for fuel cells applications [1]. Moreover, having physical properties similar to LPG, supply of DME to the end users could be well accomplished using the existing LPG infrastructure with minor investment costs.

Traditionally, DME is produced by a two-step process involving the conversion of syngas to methanol in the presence of a $\mathrm{Cu}-\mathrm{ZnO}$-based catalyst and its subsequent dehydration to DME on a solid acid catalyst [3]. Though typically syngas used in the methanol synthesis step is produced from fossil resources (natural gas, coal, and oil), the utilization of syngas derived from biomass via gasification has recently attracted considerable interest [4]. In the conventional process, the conversion of syngas to methanol is limited by the thermodynamic equilibrium requiring high pressures and low temperatures in order to attain reasonable per-pass conversions. Lately, a new one-step

process for the direct conversion of syngas to DME (so-called STD process) has received much attention as it is thermodynamically and economically more advantageous than the traditional two-step technology $[5,6]$. The key reactions in STD are the hydrogenation of $\mathrm{CO}$ and $\mathrm{CO}_{2}$ (typically present in biomass-derived syngas) to 
methanol, the methanol dehydration to DME, and the water-gas-shift reaction (WGSR) according to the chemical equations (1) to (4) below:

$$
\begin{aligned}
& 2 \mathrm{CO}+4 \mathrm{H}_{2} \leftrightarrow 2 \mathrm{CH}_{3} \mathrm{OH} \\
& \mathrm{CO}_{2}+3 \mathrm{H}_{2} \leftrightarrow \mathrm{CH}_{3} \mathrm{OH}+\mathrm{H}_{2} \mathrm{O} \\
& 2 \mathrm{CH}_{3} \mathrm{OH} \leftrightarrow \mathrm{CH}_{3} \mathrm{OCH}_{3}+\mathrm{H}_{2} \mathrm{O} \\
& \mathrm{H}_{2} \mathrm{O}+\mathrm{CO} \leftrightarrow \mathrm{H}_{2}+\mathrm{CO}_{2}
\end{aligned}
$$

The concerted action of this set of reactions leads to a synergetic effect by which the methanol formed from $\mathrm{CO} / \mathrm{CO}_{2}$ hydrogenation is consumed through reaction (3) shifting the chemical equilibrium for the methanol synthesis reactions (1) and (2) to the right-hand side allowing high per-pass $\mathrm{CO}$ conversions to be attained. Moreover, the water formed in reactions (2) and (3) reacts with CO through the WGSR to produce $\mathrm{CO}_{2}$ and $\mathrm{H}_{2}$ (equation 4) which are reactants for the methanol synthesis. It is clear, thus, that catalysts employed in the STD process should be able to efficiently catalyze both the methanol synthesis and the methanol dehydration reactions. This can be accomplished by using dual-function composite or hybrid catalysts typically comprising a $\mathrm{Cu}-\mathrm{ZnO}$-based methanol synthesis catalyst and a solid acid, most often $\gamma-\mathrm{Al}_{2} \mathrm{O}_{3}$ $[7,8,9,10,11]$ or HZSM-5 zeolite $[12,13,14,15,16,17,18]$, as the dehydration function. It is generally admitted that HZSM-5 is more suitable than $\gamma-\mathrm{Al}_{2} \mathrm{O}_{3}$ as the dehydration component in bifunctional STD catalysts as the former exhibits much higher activity at moderate reaction temperatures $\left(\mathrm{ca} .240-280^{\circ} \mathrm{C}\right.$ ) which are thermodynamically more favorable for the methanol synthesis step [9,19]. Furthermore, water formed by methanol dehydration (and $\mathrm{CO}_{2}$ hydrogenation if present in the feed) strongly adsorbs on the Lewis acid sites of $\gamma-\mathrm{Al}_{2} \mathrm{O}_{3}$ inhibiting DME formation while the effect of water is 
much less significant for HZSM-5 owing to its more hydrophobic character and predominance of Brønsted-type acidity $[9,14,19]$.

Since the methanol synthesis over Cu-type catalysts is a well established and quite optimized technology, much of the efforts in the researches concerning the STD process have been devoted to the study of the methanol dehydration function. The general conclusion one may withdraw from these previous studies is that the acid property of the dehydration component plays a crucial role in determining the overall CO conversion rate, DME selectivity, and catalyst durability. Therefore, a deep understanding of the role of the different types of acid sites (either Brønsted or Lewis) present in the dehydration catalyst, their amount, and strength becomes essential in order to perform the STD process in the most efficient way. Unfortunately, the previous literature in this respect is somehow unclear due to the higher complexity of the STD process and catalyst composition with respect to the simpler methanol dehydration system which has led, in fact, to some contradictory or ambiguous conclusions. In many occasions it is observed that when the dehydration function is composed by strong solid acids, such as H-zeolites (particularly those with lower $\mathrm{Si} / \mathrm{Al}$ ratio) or sulfated-zirconia, the overall STD reaction becomes controlled by the methanol synthesis step leading to a lack of correlation between the acid property of the methanol dehydration function and the CO conversion rate or DME yield $[10,13,15,17,18,20,21,22]$. In this situation the methanol formed on the Cu-based catalyst is rapidly consumed by the acid dehydration catalyst yielding a product composition close to that predicted by the equilibrium concentration at the employed STD conditions. Moreover, even under conditions where the STD reaction is controlled by the methanol synthesis, discrepancies can also be found in the literature regarding the influence of acidity on the $\mathrm{CO}$ conversion or product selectivity. For instance, while Mao et al. [18] postulated that under these 
conditions the acidity only influences DME selectivity without affecting CO conversion, Wang et al. [17] observed that increasing the amount of acid sites in the hybrid catalyst produced a decrease in $\mathrm{CO}$ conversion with no appreciable changes in DME selectivity. In contrast to the above two works, other authors did not find significant changes in CO conversion and DME selectivity with the acidity of the dehydration catalyst when the overall STD process is governed by the methanol synthesis step $[13,20]$.

A lack of consensus is also evidenced from the previous STD literature as regards to the requirements in terms of type of acid sites and acid strength to effectively producing DME. As to the nature of the acid sites (a mechanistic discussion is out of the scope of the present work), Sarkadi-Pribóczki et al. [23] concluded by applying a radioisotope method that Lewis acid sites (in fact Lewis acid-base pairs present on the alumina surface) are active only for methanol dehydration to form DME, while Brønsted acid sites as those associated to framework Al in zeolites are required for the formation of undesired hydrocarbons. Similarly, by comparing silica-alumina catalysts with different silica contents and thus with distinct Brønsted/Lewis acid ratios, Takeguchi et al. concluded that Lewis acid-base pairs are the major active sites for the methanol-to-DME reaction, whereas strong Brønsted acid sites in HZSM-5 favor the consecutive conversion of DME to hydrocarbons [9]. By contrast, Xia et al. suggested that extralattice Al species, which are typically associated to the development of Lewistype acidity in zeolites, do promote the formation of hydrocarbons during the STD reaction [21]. In preceding studies, however, evidences were presented that Lewis acid sites in HZSM-5 are at the origin of catalytic activity in the conversion of methanol to DME [24]. By studying the effect of water addition on the methanol dehydration activity over different solid acids, Xu et al. concluded that both Brønsted and Lewis 
acid sites in HZSM-5 are active for DME formation, though obviously the former ones generally present in greater amounts in zeolites do contribute the most to the overall dehydration activity [19]. Mao et al., on the other hand, did not find a direct relationship between the activity for methanol dehydration and the nature of the acid sites on HZSM-5 modified by basic $\mathrm{MgO}$ species and proposed a mechanism for DME formation involving both acidic and basic sites [18]. Quite often the contribution of different type of acid sites could not be assessed simply because the acidity of the dehydration catalysts was exclusively characterized by using the $\mathrm{NH}_{3}-\mathrm{TPD}$ technique $[10,13,15,17]$ which, obviously, is not able to discriminate between Brønsted and Lewis acid sites. Concerning the acid strength, many authors concluded (mostly based on $\mathrm{NH}_{3}$-TPD profiles) that acid sites of intermediate (or moderate) strength are the most desirable from the viewpoint of DME selectivity, while strong acid sites do promote the formation of by-products in detriment of DME $[9,10,19]$. Others, however, stated that acid sites of relatively weak strength (again based on $\mathrm{NH}_{3}-\mathrm{TPD}$ results) can also efficiently convert methanol to DME [18] or even that weak acid sites are more effective for the selective DME synthesis [12,25]. Just on the opposite side, Kim et al. [13] associated the dehydration activity of Na- and H-ZSM-5 samples to the presence of very strong acid sites characterized by $\mathrm{NH}_{3}$ desorption temperatures above $500^{\circ} \mathrm{C}$ (socalled $\gamma$ sites).

It is apparent from the above discussions that more systematic works are required in order to gaining more insights into the influence of the acid property of the dehydration catalyst, hopefully differentiating by type of acid site, density, and strength, on the activity and selectivity during the STD process. For this purpose, in the present investigation we have prepared a series of hybrid CuZnAl/ZSM-5 catalysts in where the acid properties of the zeolite dehydration component were systematically varied by 
submitting a commercial HZSM-5 sample to different post-synthesis treatments. The acid properties of the zeolite samples have been exhaustively measured by applying both $\mathrm{NH}_{3}$-TPD and FTIR-pyridine techniques. The catalytic behavior for the STD reaction of the prepared $\mathrm{CuZnAl} / \mathrm{ZSM}-5$ hybrids was determined under conditions where the overall reaction is either controlled by the methanol dehydration step, as confirmed by independent methanol dehydration experiments at the applied STD temperature of $260^{\circ} \mathrm{C}$, or by the methanol synthesis reaction.

\section{EXPERIMENTAL}

\subsection{Preparation of catalysts}

\subsubsection{Cu-based methanol synthesis catalyst}

The $\mathrm{CuO}-\mathrm{ZnO}-\mathrm{Al}_{2} \mathrm{O}_{3}$ (abbreviated as $\mathrm{CZA}, \mathrm{Cu}: \mathrm{Zn}: \mathrm{Al}$ atomic ratio= 6:3:1) methanol synthesis catalyst precursor was prepared by coprecipitation following the optimized methodology described in [26]. Briefly, an aqueous solution of the metal nitrates $\left[\mathrm{Cu}\left(\mathrm{NO}_{3}\right)_{2}(0.6 \mathrm{M}), \mathrm{Zn}\left(\mathrm{NO}_{3}\right)_{2}(0.3 \mathrm{M})\right.$, and $\left.\mathrm{Al}\left(\mathrm{NO}_{3}\right)_{3}(0.1 \mathrm{M})\right]$ and an aqueous

solution of $\mathrm{Na}_{2} \mathrm{CO}_{3}(1 \mathrm{M})$ as precipitating agent were simultaneously added at a constant flow rate of $5 \mathrm{~mL} / \mathrm{min}$ to a glass beaker kept under stirring at a temperature of $70^{\circ} \mathrm{C}$ and a constant $\mathrm{pH}$ of $7.0 \pm 0.1$ (measured through a Mettler Toledo $\mathrm{pH}$ meter). Once the addition of the metal nitrates solution was completed the suspension was aged for $1 \mathrm{~h}$ at the same temperature while keeping constant the $\mathrm{pH}$ at 7.0 through the controlled addition of the metal nitrates or sodium carbonate solutions. Subsequently, the precipitate was filtered, exhaustively washed with deionized water, dried in an oven at $100^{\circ} \mathrm{C}$ for $12 \mathrm{~h}$, and finally calcined under air flow $\left(25 \mathrm{~mL} \cdot \mathrm{min}^{-1} \cdot \mathrm{g}^{-1}\right)$ at $300^{\circ} \mathrm{C}$ for $3 \mathrm{~h}$ yielding the oxide CZA catalyst precursor. 


\subsubsection{Zeolite dehydration catalysts}

The starting ZSM-5 zeolite was a commercial sample in its protonic form (CBV3020, Zeolyst Int., denoted here as HZ) with Si/Al atomic ratio of 16. Aliquots of this sample were submitted to repeated ionic exchanges $\left(80^{\circ} \mathrm{C}, 12 \mathrm{~h}\right.$, liquid/solid ratio of $10 \mathrm{~mL} / \mathrm{g})$ with aqueous solutions of either $\mathrm{NaNO}_{3}$ or $\mathrm{Co}\left(\mathrm{NO}_{3}\right)_{2}(0.1-1.0 \mathrm{M})$ so as to attain the desired exchange degree of $\mathrm{H}^{+}$by $\mathrm{Na}^{+}$or $\mathrm{Co}^{2+}$ ions. After each ionic exchange the solids were washed with a large excess of deionized water and dried at $100^{\circ} \mathrm{C}$ for 12 h. The exchanged zeolites were designated as $\mathrm{M} x \mathrm{HZ}(\mathrm{M}=\mathrm{Na}, \mathrm{Co})$, where $x$ is 100 times the $\mathrm{M} / \mathrm{Al}$ atomic ratio, as determined by ICP-OES (vide infra).

Another aliquot of the commercial HZ sample was submitted to an acid leaching treatment with an aqueous $1 \mathrm{M} \mathrm{HCl}$ solution at $100^{\circ} \mathrm{C}$ (reflux) for $12 \mathrm{~h}$ (liquid/solid ratio of $10 \mathrm{~mL} / \mathrm{g}$ ), followed by exhaustive washing with deionized water and drying at $100^{\circ} \mathrm{C}$ for $12 \mathrm{~h}$. The acid-treated zeolite was labeled as HZA.

Finally, silicalite-1 (the all-silica counterpart of ZSM-5) was synthesized following the procedure reported in [27]. Materials used for the synthesis were tetraethylorthosilicate (TEOS, Aldrich, $\geq 99 \%$ ), tetrapropylammonium hydroxide (TPAOH, 40 wt $\%$ aqueous solution, Alfa Aesar), $\mathrm{NaOH}$ (Aldrich, $\geq 98 \%$ ), and MilliQ water. The reagents were mixed in the a TPAOH:NaOH:TEOS: $\mathrm{H}_{2} \mathrm{O}$ molar ratio of 9:0.16:25:495 and stirred overnight to ensure complete hydrolysis of TEOS. Then, the obtained clear solution was transferred to a Teflon-lined autoclave and heated in an oven at $160^{\circ} \mathrm{C}$ for $48 \mathrm{~h}$ under static conditions. The obtained solid was recovered after three cycles of centrifuging and washing with deoinized water until a neutral $\mathrm{pH}$ was observed in the washing waters. Subsequently, the solid was dried in an oven at $100^{\circ} \mathrm{C}$ 
for $12 \mathrm{~h}$ and finally calcined in air $(100 \mathrm{~mL} / \mathrm{min})$ at $600^{\circ} \mathrm{C}$ for $10 \mathrm{~h}$ to remove the organic material. The obtained silicalite-1 sample was designed as S1, and was used here as reference for assessing the STD behavior of an acid site-free hybrid CZA/zeolite catalyst.

\subsubsection{Hybrid CZA/zeolite catalysts}

Two series of hybrid catalysts with CZA/zeolite mass ratios of 2:1 and 10:1, respectively, were prepared by physically mixing the calcined pre-pelletized $(0.25-0.42$ mm sieve fraction) CZA and zeolite components. Additionally, a series of hybrids with a CZA/zeolite mass ratio of 2:1 was also prepared by careful grinding the individual powders in an agate mortar followed by pelletizing $(0.25-0.42 \mathrm{~mm})$ the homogenous powder mixture. The series of hybrids were denoted as $\mathrm{CZA} / Z(X: Y) \mathrm{P}$, where $Z$ is the notation applied for the ZSM-5 samples as described in section 2.1.2, $X: Y$ is the $\mathrm{CZA} /$ zeolite mass ratio, and $P$ is $\mathrm{M}$ or $\mathrm{G}$ depending on the method used for preparing the physical mixtures $(M=$ mixture of pre-pelletized solids; $G=$ grinding of powders followed by pelletizing). For instance, the hybrid denoted as CZA/Co20HZ(2:1)M refers to the catalyst prepared by mixing the pellets of CZA and Co20HZ zeolite (exchanged with $\left.\mathrm{Co}^{2+}, \mathrm{Co} / \mathrm{Al}=0.20\right)$ in a $\mathrm{CZA} /$ zeolite mass ratio of $2: 1$.

\subsection{Characterization techniques}

Phase purity of the synthesized silicalite-1 zeolite and crystallinity of the modified ZSM-5 samples relative to the parent commercial HZ zeolite (taken as $100 \%$ crystalline) were assessed by powder X-ray diffraction (XRD) in a Philips X'Pert diffractometer equipped with a graphite monochromator operating at $40 \mathrm{kV}$ and $45 \mathrm{~mA}$ 
using nickel-filtered $\mathrm{Cu} \mathrm{K} \alpha$ radiation $(\lambda=0.1542 \mathrm{~nm})$. XRD was also applied to assess the crystalline phases present in the calcined CZA methanol synthesis catalyst precursor. The chemical composition ( $\mathrm{Si} / \mathrm{Al}$ and $\mathrm{M} / \mathrm{Al}$ atomic ratios, $\mathrm{M}=\mathrm{Na}, \mathrm{Co}$ ) of the ZSM-5 samples and metal contents in the CZA component were determined by Inductively Coupled Plasma-Optical Emission Spectroscopy (ICP-OES) in a Varian 715-ES apparatus after dissolution of the solids in an acid mixture of $\mathrm{HNO}_{3}: \mathrm{HF}: \mathrm{HCl}$ in a 1:1:3 volume ratio. Textural properties for the zeolites and CZA precursor were derived from the respective $\mathrm{N}_{2}$ adsorption isotherms recorded at $-196^{\circ} \mathrm{C}$ on an ASAP2000 (Micromeritics) equipment. Prior to the measurements the samples (ca. $200 \mathrm{mg}$ ) were pretreated at $200^{\circ} \mathrm{C}(\mathrm{CZA})$ or $400^{\circ} \mathrm{C}$ (zeolites) and vacuum overnight. The lower degassing temperature applied for the CZA component is advised in order to avoid structural changes in the $\mathrm{Cu}$-based catalyst that are typically observed at temperatures above $300^{\circ} \mathrm{C}[28,29]$. The specific surface areas were obtained using the BET model and the micropore volume by applying the $t$-plot approach.

The reduction behavior of the calcined CZA precursor was studied by $\mathrm{H}_{2}-\mathrm{TPR}$ in a Micromeritics Autochem 2910 equipment. About $30 \mathrm{mg}$ of sample were initially flushed with Ar at room temperature for $30 \mathrm{~min}$. Then, the gas was switched to the reductive mixture of $10 \mathrm{vol} \% \mathrm{H}_{2}$ in $\mathrm{Ar}$ (flow rate $=50 \mathrm{~mL} / \mathrm{min}$ ) and the temperature linearly increased from ambient till $600^{\circ} \mathrm{C}$ at a heating rate of $10^{\circ} \mathrm{C} / \mathrm{min}$. Water formed in the reduction was retained in a 2-propanol/ $\mathrm{N}_{2(1)}$ trap and the $\mathrm{H}_{2}$ consumption rate was monitored in a thermal conductivity detector (TCD) previously calibrated using the reduction of $\mathrm{CuO}$ as a reference.

${ }^{27}$ Al MAS NMR (Magic-Angle Spinning Nuclear Magnetic Resonance) spectra were recorded at room temperature in a Bruker AV-400 WB spectrometer operating at 104.2 $\mathrm{MHz}$ and equipped with a $4 \mathrm{~mm}$ Bruker BL4mm probe. Samples were packed 
into zirconia rotors and spun at the magic angle spinning (MAS) at $10 \mathrm{kHz}$. The ${ }^{27} \mathrm{Al}$ spectra were acquired with pulses of $0.5 \mu$ s corresponding to a flip angle of $\pi / 18$ and chemical shifts were referred to a $0.1 \mathrm{M}$ aqueous solution of $\mathrm{Al}\left(\mathrm{NO}_{3}\right)_{3}$.

The acid properties of the zeolites were assessed by both FTIR-pyridine and $\mathrm{NH}_{3}$-TPD techniques. FTIR spectra of adsorbed pyridine were recorded in a Nicolet 710 FTIR apparatus for self-supported wafers $\left(10 \mathrm{mg} / \mathrm{cm}^{2}\right)$ previously pretreated at $400^{\circ} \mathrm{C}$ overnight under dynamic vacuum of $10^{-4} \mathrm{~Pa}$. Afterwards, $1.8 \cdot 10^{3} \mathrm{~Pa}$ of pyridine were admitted to the IR cell at room temperature and after equilibration the samples were degassed for $1 \mathrm{~h}$ at 250,350 , and $400^{\circ} \mathrm{C}$. After each desorption step, the spectrum was recorded at room temperature and the background subtracted. The amounts of Brønsted and Lewis acid sites were determined from the integrated areas of the bands at ca. 1545 and $1450 \mathrm{~cm}^{-1}$, respectively, using the extinction coefficients reported by Emeis [30]. In turn, $\mathrm{NH}_{3}$-TPD profiles were obtained in an Autochem 2910 equipment (Micromeritics) coupled with a quadrupole mass spectrometer (OmniStar from Balzers Intruments). Prior to ammonia adsorption, ca. $100 \mathrm{mg}$ of sample were pretreated at $450^{\circ} \mathrm{C}$ for 30 minutes in $\mathrm{O}_{2}$, then for 15 minutes in $\mathrm{Ar}$, and finally for 15 minutes in He. Subsequently, calibrated pulses of $\mathrm{NH}_{3}$ were introduced at $175^{\circ} \mathrm{C}$ until saturation of the sample and the chemisorbed ammonia subsequently desorbed by heating from $175^{\circ} \mathrm{C}$ to $900^{\circ} \mathrm{C}$ at a heating rate of $10^{\circ} \mathrm{C} / \mathrm{min}$. The $\mathrm{m} / \mathrm{z}=17$ signal in the coupled mass spectrometer was used to obtain the corresponding $\mathrm{NH}_{3}$ desorption profiles.

\subsection{Catalytic experiments}

\subsubsection{Methanol dehydration}

The methanol dehydration activity for the parent and modified ZSM-5 samples was determined in a continuous-flow fixed-bed quartz reactor at atmospheric pressure 
and $260^{\circ} \mathrm{C}$ (the same reaction temperature applied for the STD experiments, vide infra). Typically, $15-30 \mathrm{mg}$ of zeolite $(0.25-0.42 \mathrm{~mm}$ pellet size) were diluted with $2 \mathrm{~mL}$ of inert $\mathrm{SiC}$ particles $(0.6-0.8 \mathrm{~mm}$ sieve fraction) and then loaded to the reactor. Prior to feeding methanol the catalyst was routinely activated in situ under flowing nitrogen (60 $\mathrm{mL} / \mathrm{min}$ ) at $300^{\circ} \mathrm{C}$ for $1 \mathrm{~h}$. Then, the reactor temperature was lowered to $260^{\circ} \mathrm{C}$ and the $\mathrm{N}_{2}$ flow replaced by a mixture of $\mathrm{N}_{2}$ and $\mathrm{MeOH}$ vapor $\left(\mathrm{MeOH}: \mathrm{N}_{2}\right.$ molar ratio= 20:80, total flow rate $=150 \mathrm{~mL} / \mathrm{min}$ ). The amount of zeolite in the reactor was adjusted so as to obtain initial methanol conversions $<30 \%$. The composition of the reactor effluent was determined on line at regular time intervals in a gas chromatograph (HP-GC6890) equipped with a TBR-5 capillary column and a flame ionization detector (FID). Initial methanol dehydration rates were obtained by extrapolating the rate-TOS (time-onstream) curves at $\mathrm{TOS}=0$.

\subsubsection{Syngas-to-DME (STD)}

STD runs were performed in a 310 stainless-steel fixed-bed reactor $(40 \mathrm{~cm}$ length, $10 \mathrm{~mm}$ i.d.) charged with $1.0 \mathrm{~g}$ of hybrid catalyst $(0.25-0.42 \mathrm{~mm})$ previously diluted with $\mathrm{SiC}$ granules $(0.6-0.8 \mathrm{~mm})$ to achieve a total bed volume of $10 \mathrm{~mL}$. Before reaction, the catalyst was exposed to a diluted $\mathrm{H}_{2}$ stream $\left(5 \mathrm{vol} \% \mathrm{H}_{2}\right.$ in $\mathrm{N}_{2}$ ) at $245^{\circ} \mathrm{C}$ for $10 \mathrm{~h}$ in order to reduce $\mathrm{CuO}$ in the $\mathrm{CZA}$ catalyst to its active metallic $\mathrm{Cu}$ state. The gaseous feed stream consisted of a mixture of 90 vol\% syngas (molar composition: $66 \% \mathrm{H}_{2} / 30 \% \mathrm{CO} / 4 \% \mathrm{CO}_{2}$ ) and $10 \mathrm{vol} \% \mathrm{Ar}$ (used as internal standard for GC analyses) premixed in a single pressurized cylinder, and the STD reaction was carried out at $260^{\circ} \mathrm{C}, 4.0 \mathrm{MPa}$, and space velocity of $1700 \mathrm{~mL}_{\text {syngas }} /\left(\mathrm{g}_{\text {cat }} \cdot \mathrm{h}\right)$ referred to the mass of CZA component. Reaction products were analyzed on line in a Varian 450-GC gas chromatograph equipped with a TCD for the separation and quantification of $\mathrm{CO}, \mathrm{CO}_{2}$, 
and Ar, and a FID for the analysis of oxygenates (MeOH, DME) and hydrocarbons. To avoid any product condensation during the reaction, the lines from the reactor to the injection valve of the gas chromatograph were kept at $180^{\circ} \mathrm{C}$ during the entire run. Product yields and selectivities are given on a carbon basis. Carbon mass balances for the reported STD runs were $100 \pm 2 \%$.

\section{RESULTS AND DISCUSSION}

\subsection{Characterization of materials}

\subsubsection{Methanol synthesis component (CZA)}

The metal $\mathrm{Cu} / \mathrm{Zn} / \mathrm{Al}$ atomic ratio of the $\mathrm{CZA}$ oxide precursor as determined by ICP-OES was 6.0/2.9/1.0, which practically matched the intended 6/3/1 ratio according to the precursor solution concentrations. The X-ray diffractogram of the calcined CZA precursor is shown in Fig. S1 (Supplementary Material). There, the presence of $\mathrm{CuO}$ and $\mathrm{ZnO}$ crystalline phases with some overlapping peaks can be readily inferred by comparison with the characteristic diffraction patterns for the pure crystalline oxides which are also shown in Fig. $\mathrm{S} 1$ as a guide. The mean crystallite size of the $\mathrm{CuO}$ phase estimated by the Scherrer equation from the non-overlapped $\mathrm{CuO}$ reflection at ca. $38.8^{\circ}$ was $5.4 \mathrm{~nm}$. No peaks related to $\mathrm{Al}_{2} \mathrm{O}_{3}$ were observed, indicating that this phase should be present as very small XRD-silent crystallites or as an amorphous phase, in agreement with previous studies [26]. Furthermore, no diffraction peaks associated to $\mathrm{Cu} / \mathrm{Zn}$ hydroxycarbonate residues were evidenced in the pattern of the calcined precursor, though they were clearly observed in the XRD of the dried solid (not shown), indicating that these phases were almost totally decomposed during the calcination stage. The BET surface area of the calcined precursor was $98 \mathrm{~m}^{2} / \mathrm{g}$, a value that falls within the expected range of $90-110 \mathrm{~m}^{2} / \mathrm{g}$ according to the methodology and composition employed in its 
preparation [26]. Finally, the $\mathrm{H}_{2}$-TPR profile of the methanol synthesis catalyst showed a single and relatively sharp reduction feature peaking at $205^{\circ} \mathrm{C}$ (see Fig. S2 in Supplementary Material) that is related to the reduction of $\mathrm{Cu}^{2+}$ in the oxide phase to metallic $\mathrm{Cu}$. In fact, by considering the $\mathrm{Cu}$ content in the catalyst (from ICP-OES), the experimental $\mathrm{H}_{2}$ consumption for this peak coincided (within experimental error) with the theoretical value expected for the stoichiometric reduction of $\mathrm{Cu}^{2+}$ to $\mathrm{Cu}^{0}$. Similar to our results, the most active CZA catalysts reported in the comprehensive study from Schüth and coworkers displayed a single reduction feature in the narrow $220-240^{\circ} \mathrm{C}$ range, while less active samples prepared under acidic conditions featured higher reduction temperatures owing to the presence of less favorable larger crystallites [26]. It may be inferred, thus, from these results that almost all the $\mathrm{Cu}$ species will be in its active metallic state after the $\mathrm{H}_{2}$-reduction treatment at $245^{\circ} \mathrm{C}$ performed in situ prior to catalysis.

\subsubsection{Methanol dehydration component (ZSM-5 zeolite)}

The XRD patterns for the commercial HZSM-5 sample (HZ), silicalite-1 (S1), representative samples of the exchanged series (Na39HZ, Co20HZ), and the acidtreated zeolite (HZA) are presented in Fig. S3 (Supplementary Material). As seen there, the calcined S1 zeolite synthesized in this work showed high crystallinity and phase purity. It is also seen by comparing the intensity of the main MFI-phase reflections that a high crystallinity was retained upon the ionic exchanges and the acid leaching treatment. The calculated relative XRD crystallinities (considering the untreated commercial sample as $100 \%$ crystalline), the results from chemical analysis, and the textural properties of the ZSM-5 samples are given in Table 1. The high degree of crystallinity retained for the modified samples $(87-99 \%)$ is consistent with the small 
relative decrease in BET surface area and micropore volume noticed after the treatments (less than $15 \%$ in the worse case).

As also seen in Table 1, submitting the parent $\mathrm{HZ}$ zeolite to ionic exchanges with $\mathrm{Na}^{+}$and $\mathrm{Co}^{2+}$ cations did not alter the bulk $\mathrm{Si} / \mathrm{Al}$ ratio, while a slight increase from 16 to 18 was noticed for the acid-treated HZA sample as indicative of a partial extraction of $\mathrm{Al}$ from the solid during the treatment. The coordination state of $\mathrm{Al}$ species in the parent and treated samples was studied by ${ }^{27}$ Al MAS NMR spectroscopy, and the spectra for selected samples are depicted in Fig. 1. For all samples most of the Al species were in tetrahedral coordination $\left(\mathrm{Al}^{\mathrm{IV}}, \delta \approx 53 \mathrm{ppm}\right)$ and thus occupying framework positions in the zeolite lattice, though the presence of penta $\left(\mathrm{Al}^{\mathrm{V}}\right.$, broad signal at $\delta \approx 30 \mathrm{ppm})$ and hexacoordinated $\left(\mathrm{Al}^{\mathrm{VI}}, \delta \approx-1 \mathrm{ppm}\right)$ extraframework $\mathrm{Al}$ species (generally denoted as EFAL) was also apparent. In general, the ionic exchanges and the acid treatment did not produce major changes in the coordination state of $\mathrm{Al}$ species. However, a closer inspection to the spectra revealed that the changes, though subtle, were more evident for the acid-treated HZA zeolite. In this case, the $\left(\mathrm{Al}^{\mathrm{V}}+\mathrm{Al}^{\mathrm{VI}}\right) / \mathrm{Al}^{\mathrm{IV}}$ intensity ratio was slightly decreased as compared to the untreated $\mathrm{HZ}$ zeolite and the tetrahedral peak became narrower and more symmetrical, suggesting that the acid treatment removed part of both framework and extraframework Al species.

The consequences of the ionic exchanges and acid treatment on the acid properties of the ZSM-5 zeolite will be analyzed on the bases of FTIR-pyridine and $\mathrm{NH}_{3}$-TPD results. The amounts of Brønsted and Lewis acid sites at increasing pyridine desorption temperatures $\left(250^{\circ} \mathrm{C} \rightarrow 400^{\circ} \mathrm{C}\right)$ for the parent and modified zeolites are presented in Table 2. The corresponding FTIR spectra in the pyridine vibration region after desorption at $250^{\circ} \mathrm{C}$ are shown in Fig. 2. Most of the acid sites in the parent $\mathrm{HZ}$ sample were of the Brønsted type (Brønsted/Lewis ratio $=4.5$ at $250^{\circ} \mathrm{C}$ desorption 
temperature) concurring with the predominance of tetrahedral Al species, as previously discussed on the basis of ${ }^{27} \mathrm{Al}$ MAS NMR results. As expected, for both the NaxHZ and CoxHZ series the amount of total Brønsted acid sites (after desorbing pyridine at $250^{\circ} \mathrm{C}$, B250) decreased with increasing the cation content. In fact, a fairly good linear correlation $\left(\mathrm{R}^{2}>0.99\right)$ between $\mathrm{B} 250$ and the atomic $\mathrm{M} / \mathrm{Al}$ ratio becomes apparent in Fig. 3a. The slope of the linear fitting for the Co-exchanged series $\left(b_{C o}=-820\right)$ is almost twice that of the Na-exchanged one $\left(b_{\mathrm{Na}}=-450\right)$, which nicely agrees with the fact that each $\mathrm{Co}^{2+}$ cation should replace two $\mathrm{H}^{+}$while obviously the exchange stoichiometry should be $1: 1$ in the case of $\mathrm{Na}^{+}$. It is clear that each divalent $\mathrm{Co}^{2+}$ cation should be balancing two nearby $\mathrm{AlO}_{4}{ }^{-}$tetrahedra in the ZSM-5 lattice, a fact that was shown to be favorable for low $\mathrm{Si} / \mathrm{Al}$ ratio $\mathrm{MFI}$ samples (as that used here with $\mathrm{Si} / \mathrm{Al}=16$ ) where the distances between $\mathrm{AlO}_{4}{ }^{-}$tetrahedra are relatively short [31]. In addition, the cationic exchanges were seen to reduce the average strength of the remaining Brønsted acid sites as compared to the original $\mathrm{HZ}$ sample, the effect being more marked for the Naexchanged series, as inferred from the change in the B400/B250 ratio with M/A1 in Fig. 3b. On the other hand, the change in Lewis acidity showed different trends depending on the nature of the exchange cation. Thus, the density of Lewis acid sites (after desorbing pyridine at $250^{\circ} \mathrm{C}, \mathrm{L} 250$ ) decreased with the partial exchange of $\mathrm{H}^{+}$with $\mathrm{Na}^{+}$ while the reverse trend was observed upon the exchange with $\mathrm{Co}^{2+}$ cations (Fig. 3c). In this later case, the amount of Lewis acid sites progressively raised with the amount of exchanged $\mathrm{Co}^{2+}$ cations whereas for the $\mathrm{Na}^{+}$-exchanged series the decrease was more notorious at low $\mathrm{M} / \mathrm{Al}$ ratios. Furthermore, the strength of the Lewis acid sites slightly increased or remained similar to that in the parent $\mathrm{HZ}$ zeolite for the $\mathrm{Na}^{+}$-exchanged samples but became clearly lower for the CoxHZ series, as inferred from the respective L400/L250 ratios depicted in Fig. 3d. The increase in the concentration of Lewis acid 
sites observed for the CoxHZ series can be attributed to the presence of $\mathrm{Co}^{2+}$ ions in exchange positions of the ZSM-5 zeolite [32]. Indeed, as evidenced in the FTIRpyridine spectra of $\mathrm{CoxHZ}$ samples shown in Fig. $2, \mathrm{Co}^{2+}$ species located at exchange positions generated new Lewis acid sites characterized by a pyridine adsorption band at $1452 \mathrm{~cm}^{-1}$ different from the typical band at $1456 \mathrm{~cm}^{-1}$ associated to EFAL species, in accord with previous observations [31]. The shift towards lower wavenumbers can be taken as indicative of a lower acid strength of the Lewis acid sites associated to the exchanged $\mathrm{Co}^{2+}$ relative to those linked to EFAL. The Lewis acid band at $1612 \mathrm{~cm}^{-1}$ clearly distinguished in the FTIR-pyridine spectra of the CoxHZ samples (Fig. 2) has also been linked to the presence of $\mathrm{Co}^{2+}$ ions in exchange positions within the zeolite channels [31]. The fact that its intensity increases with the degree of exchange (i.e. $\mathrm{Co} / \mathrm{Al}$ ratio) reinforces this assignment. In the case of $\mathrm{Na}^{+}$-exchanged samples, two factors might contribute to the observed decrease in the amount of pyridine interacting with Lewis acid sites with respect to the parent $\mathrm{HZ}$ zeolite (Table 2, Fig. 3c). On one hand, part of cationic-type EFAL species responsible for the strong Lewis acidity in the original zeolite could have been exchanged by $\mathrm{Na}^{+}$ions and, on the other hand, it is likely that the Lewis acid sites associated to the exchanged $\mathrm{Na}^{+}$ions are not strong enough to coordinate pyridine at a desorption temperature of $250^{\circ} \mathrm{C}$. This aspect will be further discussed on the light of $\mathrm{NH}_{3}$-TPD results.

In the case of the HZA sample, results in Table 2 show that the acid treatment barely modified the amount and strength distribution of the Brønsted acid sites. By contrast, the total amount of Lewis acid sites (i.e. those retaining pyridine at $250^{\circ} \mathrm{C}$, L250) was largely reduced by ca. $85 \%$ with respect to the parent zeolite (from 56 to 8 $\mu \mathrm{mol} / \mathrm{g}$ ) which can be related to the selective elimination of EFAL species during the acid treatment. In fact, removal of both framework and extraframework Al species 
during the acid treatment was suggested on the basis of ${ }^{27} \mathrm{Al}$ MAS NMR characterization (Fig. 1). At this point, it should be mentioned that the fact that the Brønsted acidity was hardly modified upon the acid treatment might be the consequence of the opposing effects caused by the partial removal of both framework and cationictype extraframework (neutralizing part of the $\mathrm{H}^{+}$) Al species.

Finally, the changes in the strength distribution of the zeolite acid sites arising from the applied treatments were also assessed from the corresponding $\mathrm{NH}_{3}$-TPD profiles depicted in Fig. 4. As seen there, no $\mathrm{NH}_{3}$ adsorption occurred on the silicalite-1 sample which resulted in a flat desorption profile, as expected from its all-silica nature. On the other hand, similar $\mathrm{NH}_{3}$ desorption profiles were found for the parent (HZ) and the acid-treated (HZA) samples, both featuring a main desorption peak at about $350^{\circ} \mathrm{C}$ attributed to relatively strong acid sites along with a lower-temperature shoulder peaking at ca. $245^{\circ} \mathrm{C}$ assigned to weak acid sites. The partial exchange of $\mathrm{H}^{+}$in $\mathrm{HZ}$ with $\mathrm{Na}^{+}$or $\mathrm{Co}^{2+}$ cations decreased the amount of strong acid sites (particularly in the case of $\mathrm{Na}^{+}$) and increased that of the weak ones. For the NaxHZ series, the weak-to-strong acid ratio significantly increased with the increase in the amount of exchanged $\mathrm{Na}^{+}$cations. These results indicate, on one hand, that $\mathrm{Na}^{+}$cations do preferentially exchange with the strongest $\mathrm{H}^{+}$in the original $\mathrm{HZ}$ zeolite and, on the other hand, that the large increase in the amount of weak acid sites $\left(\mathrm{T}_{\max }=245^{\circ} \mathrm{C}\right)$ is likely due to new weak Lewis acid sites associated to $\mathrm{Na}^{+}$cations in zeolitic exchange positions. The latter fact seems to support the hypothesis previously made that these new Lewis acid sites are too weak to coordinate pyridine at $250^{\circ} \mathrm{C}$, and thus they were not detected by FTIR-pyridine producing an apparent decrease in the density of Lewis acid sites relative to the parent zeolite. In general, $\mathrm{NH}_{3}$-TPD results were in good qualitative agreement with those derived from FTIR-pyridine. 
The following main conclusions could be withdrawn from the above acidity study by combining the FTIR-pyridine and $\mathrm{NH}_{3}$-TPD results: a) the parent $\mathrm{HZ}$ and the acid-treated HZA samples displayed equivalent density and strength distribution for Brønsted-type acid sites, the main difference being the much lower density of strong Lewis acid sites associated to EFAL species in HZA; b) the partial exchange with $\mathrm{Na}^{+}$ cations preferentially neutralized strong Brønsted acid sites in the parent $\mathrm{HZ}$ zeolite and increased the amount of Lewis acid sites related to $\mathrm{Na}^{+}$displaying much weaker strength (unable to retain pyridine at $250^{\circ} \mathrm{C}$ ) than those linked to EFAL; c) the partial

exchange with divalent $\mathrm{Co}^{+2}$ ions also reduced the amount of Brønsted acid sites, with each $\mathrm{Co}^{2+}$ replacing $2 \mathrm{H}^{+}$, and created new Lewis acid sites of an average acid strength intermediate between those linked to $\mathrm{Na}^{+}$and EFAL species; d) for both exchanged $\mathrm{Na} x \mathrm{HZ}$ and CoxHZ series the changes in Brønsted and Lewis acidity varied with the cation content (or M/Al ratio). Therefore, the set of prepared ZSM-5 zeolites, comprising from samples displaying almost exclusively Brønsted-type acidity (HZA) to others having both Brønsted and Lewis acid sites in different concentrations and relative strengths, makes it suitable to tackle the catalytic study aiming at clearing up the role of zeolite acidity in the methanol dehydration and STD reactions, as will be discussed in the following sections.

\subsection{Catalytic experiments}

\subsubsection{Influence of zeolite acidity on methanol dehydration activity}

Prior to performing the STD experiments, the methanol dehydration activity of the ZSM-5 samples was evaluated at the STD temperature $\left(260^{\circ} \mathrm{C}\right)$ as detailed in section 2.3.1. Under the employed reaction conditions, the dehydration of methanol on the zeolites produced DME with a high selectivity of above $99.95 \%$ (C-basis) irrespective 
of the zeolite acidity. The variation of the methanol dehydration rate (expressed as $\mathrm{mmol} \cdot \mathrm{g}^{-1} \cdot \mathrm{s}^{-1}$ ) with TOS for the different samples is shown in Fig. S4 (Supporting Information). In general, all samples experienced a slow deactivation process with time indicative of a very low coke formation, as expected from the high coke-resistance of the medium-pore HZSM-5 zeolite and the relatively low reaction temperature applied $\left(260^{\circ} \mathrm{C}\right)$ as compared to the higher temperatures $\left(>400^{\circ} \mathrm{C}\right)$ typically employed in methanol-to-hydrocarbons processes [33]. Anyway, to avoid any possible interference from deactivation on the comparison of zeolite activities, the initial methanol dehydration rates were obtained from the rate-TOS curves in Fig. S4. With the aim of identifying the type and strength of the zeolite acid sites involved in the methanol dehydration reaction under the studied conditions, several attempts were done to correlate the obtained initial rates with the zeolite acid properties as determined by FTIR-pyridine (Table 2). This exercise revealed that the best linear correlation coefficient $\left(\mathrm{R}^{2}\right)$ was obtained when the initial dehydration rate is plotted against the amount of strong Brønsted acid sites (i.e. those retaining pyridine at $400^{\circ} \mathrm{C}, \mathrm{B} 400$ ), as shown in Fig. 5. Similarly, Ramos et al. [20] did also find a linear correlation between the methanol dehydration rate and the density of strong Brønsted acid sites in different solid acids including HZSM-5. Nevertheless, the results in Fig. 5 clearly evidence that the zeolite submitted to acid treatment (HZA) is less active for dehydrating methanol than the parent HZ sample, despite the former has even a slightly higher density of strong Brønsted acid sites. Since, as discussed before, the main effect of the acid treatment was the selective removal of EFAL species with the concomitant decrease in the amount of EFAL-related strong Lewis acid sites, it may be readily inferred that these sites could also be active for methanol dehydration at the relatively low STD temperature of $260^{\circ} \mathrm{C}$. In this respect, previous studies did also point towards an active 
participation of zeolitic Lewis acid sites associated to non-skeletal Al species in the formation of DME from methanol in the context of methanol-to-hydrocarbons reactions [24,34]. A further consequence of the correlation depicted in Fig. 5 is that weaker Lewis acid sites as those associated to the exchanged $\mathrm{Na}^{+}$and $\mathrm{Co}^{2+}$ cations in the $\mathrm{Nax} \mathrm{HZ}$ and CoxHZ samples, respectively, do not seem to contribute appreciably to the overall zeolite dehydration activity.

According to the main reactions involved in the STD process (see Introduction), it could be expected that the higher the activity for methanol dehydration of the solid acid component the higher the driving force for $\mathrm{CO} / \mathrm{CO}_{2}$ hydrogenation would be by shifting the equilibrium of reactions (1) and (2) to the right-hand side. In other words, there should be a correspondence between the CO conversion during STD and the dehydration activity of the acid component as long as the overall STD reaction is controlled by the methanol dehydration step. In order to support this, hybrid catalysts prepared by mixing the pre-pelletized CZA and zeolite solids with a high CZA:zeolite ratio $(10: 1)$ to ensure control of the reaction by the acid function were evaluated for the STD reaction. As it can be seen in Fig. 6, a fairly nice linear correlation between the CO conversion (and DME yield) obtained in the dehydration-controlled STD experiments and the independently determined initial methanol dehydration rate is evidenced. These results show that under dehydration-controlled STD conditions the efficiency for the one-step synthesis of DME from syngas of the CZA/HZSM-5 hybrids is directly proportional to the methanol dehydration activity of the zeolite which, in turn, is mostly driven by the density of strong Brønsted acid sites with a non-negligible contribution from EFAL-related strong Lewis acid sites (if present in the zeolite).

\subsubsection{STD experiments under methanol synthesis-controlled conditions}


In order to assess whether the acidity of the HZSM-5 zeolite has an effect or not on the catalytic performance of the hybrid catalysts under conditions where the overall STD process is controlled by the methanol synthesis rate on the $\mathrm{Cu}$-based catalyst, representative hybrids comprising the parent $\mathrm{HZ}$ zeolite, the acid-treated HZA sample, and the two exchanged zeolites with the highest cation content (Na39HZ, Co20HZ) were prepared by physically mixing the pre-pelletized components in a CZA/zeolite mass ratio of 2:1. At such relatively high zeolite concentration it is expected that there is an excess of acid sites so as to rapidly dehydrate all the methanol produced making the STD process to be driven by the methanol synthesis step. The activity and selectivity of the selected hybrids during the STD reaction were followed during a total time-onstream (TOS) of about $50 \mathrm{~h}$. As shown in Fig. 7a, all the studied hybrids displayed very high and alike $\mathrm{CO}$ conversion (ca. 90\%) practically matching the theoretical equilibrium conversion of ca. $93 \%$ at the studied conditions. Moreover, no signs of deactivation were observed for any of the catalysts during the $50 \mathrm{~h}$ period of reaction. Furthermore, all catalysts displayed almost the same product selectivity (64-65\% DME, 3-4\% MeOH, $31-32 \% \mathrm{CO}_{2}$, and $<0.5 \%$ hydrocarbons, in $\% \mathrm{C}$ ) which lead to similar and constant DME yield of ca. $58 \% \mathrm{C}$, close to the estimated thermodynamic yield of ca. $63 \% \mathrm{C}$. The obvious conclusion from these results is that the zeolite acidity has no impact, at least within the studied period, on the STD performance of hybrids prepared by physically mixing the CZA and zeolite pellets (M-series) when the overall STD process is controlled by the synthesis of methanol on the CZA catalyst. In this situation, even the zeolites partially exchanged with $\mathrm{Na}^{+}$and $\mathrm{Co}^{2+}$ ions were acidic enough to bring the STD process to nearly-equilibrium conditions where near-maximum DME selectivity/yield is attained. These results concur with previous studies $[13,20]$ but differ 
from others which observed changes in CO conversion [17] or DME selectivity [18] with the zeolite acidity even under methanol synthesis-controlled conditions.

A source of the controversy in the previous literature regarding the role of zeolite acidity on the STD performance and which has been received little attention could be related to the method employed for preparing the hybrid catalysts (quite often the details of the preparation are omitted). The conclusion reached in the present work based on the results depicted in Fig. 7a is, in principle, valid for hybrids prepared by mixing the pellets of CZA (oxide precursor) and zeolite components in the desired proportion. However, a widely applied method for preparing the hybrids is by grinding the components in their powder form and then pelletizing the homogenous solid mixture to the wanted particle size $[17,18,35]$. Therefore, in order to clear up whether or not the conclusion regarding the influence of zeolite acidity on the STD behavior can be generally applied for other preparation methods, hybrids prepared by the grinding method (G-series, see section 2.1.3 of Experimental) with exactly the same composition than those obtained by mixing the pellets (M-series) were evaluated under the same experimental conditions. The results of these experiments are shown in Fig. 7b. In contrast to the high activity and stability observed for all the catalysts in the M-series (Fig. 7a), it was found that the hybrids prepared by the G-method (grinding) experienced a loss of activity with TOS. Moreover, the decay behavior was strongly dependent on the specific characteristics (i.e. acidity, presence of EFAL species and exchange cations) of the zeolite dehydration component. Thus, the stability of the Gseries catalysts decreased in the following order (only the zeolite component is mentioned): $\mathrm{HZA}>\mathrm{HZ}>\mathrm{Co} 20 \mathrm{HZ}>\mathrm{Na} 39 \mathrm{HZ}$. Additional information regarding the deactivation behavior was evidenced when comparing the evolution of product selectivity with time. Quite interestingly, the catalysts prepared by grinding and 
comprising the zeolites $\mathrm{HZ}, \mathrm{HZA}$, and Co20HZ displayed nearly the same product selectivity (64-65\% DME, 3-4\% $\mathrm{MeOH}, 31-32 \% \mathrm{CO}_{2}$, and $<0.5 \%$ hydrocarbons) as those of the M-series. Moreover, these selectivity values remained constant during the $50 \mathrm{~h}$ run (see Fig. 8a for the specific case of the Co20HZ-based catalyst) despite the CO conversion was gradually declining with TOS, particularly for the catalyst containing the Co-exchanged zeolite (Fig. 7b). The fact that the product selectivity remained constant with TOS (and with values approaching those of the thermodynamic equilibrium) suggests that for the above three catalysts within the G-series the observed decrease in $\mathrm{CO}$ conversion (much more pronounced for the catalyst comprising the Coexchanged zeolite) should be mostly related to a certain deactivation of the $\mathrm{Cu}$-based methanol synthesis component [36,37]. Interestingly, a distinct selectivity behavior with TOS was found for the catalyst comprising the Na-exchanged Na39HZ sample, as evidenced in Fig. 8b. In this case, the selectivity to $\mathrm{MeOH}$ steeply increased at the expense of DME, suggesting that for this catalyst the loss of CO conversion with TOS is likely linked with a gradual decline in the zeolite dehydration activity. The obvious conclusion in the case of hybrids prepared by grinding, contrary to that reached for the M-series catalysts (mixing method), would be that the zeolite acidity has a great impact on the activity, selectivity, and catalyst lifetime during the STD process.

The above results clearly reveal that the conclusions one may reach regarding the effect of zeolite acidity on the STD process are largely dependent on the specific method applied to preparing the physical mixtures. This fact could be one of the reasons behind the controversy found in the literature regarding the acidity issue. Though a detailed investigation of the reasons behind the worsening of the STD efficiency of hybrids prepared by grinding is out of the scope of the present study, it seems reasonable to assume that some kind of detrimental interactions between the CZA and 
zeolite components should have occurred during the catalyst preparation stage. In fact, in a very recent study [38] we observed detrimental interactions leading to a decreased $\mathrm{CO}$ conversion and DME yield in CZA/HZSM-5 hybrids (zeolite with $\mathrm{Si} / \mathrm{Al}=40$ ) prepared by grinding or slurrying the component powders prior to pelletizing as compared to the catalyst prepared by mixing the individual pellets for which no interactions are expected to occur. In that study we concluded, on the basis of EPR and FTIR-pyridine results, that the interactions comprised, at least, the partial blockage of the zeolite micropores by the CZA component and the partial exchange of zeolite protons by $\mathrm{Cu}^{2+}$ species migrated from the $\mathrm{CZA}$ catalyst, although other factors were not discarded [38]. Thus, it is likely that such kind of interactions should have also occurred in the hybrids prepared here by the grinding method. In the case of the hybrids comprising the $\mathrm{Na} 39 \mathrm{HZ}$ and $\mathrm{Co} 20 \mathrm{HZ}$ zeolites it is also reasonable to assume that part of the exchanged cations in the zeolite could migrate to the $\mathrm{Cu}$-based methanol synthesis catalyst during the grinding process affecting its catalytic performance. Indeed, the addition of small amounts of cobalt to a $\mathrm{CuO} / \mathrm{ZnO} / \mathrm{Al}_{2} \mathrm{O}_{3}$ catalyst was shown to dramatically reduce its methanol synthesis activity [39], which might support the hypothesis made before that the loss of $\mathrm{CO}$ conversion in the CZA/Co20HZ(2:1)G (Fig. $7 \mathrm{~b}$ ) is probably related to detrimental interactions affecting the activity of the

methanol synthesis catalyst. Nevertheless, further work is in progress in order to elucidate the nature of the CZA-zeolite interactions in hybrids prepared by grinding as a function of the zeolite acidity and composition.

\section{CONCLUSIONS}

A series of ZSM-5 samples with different acid properties have been prepared by submitting a commercial sample (CBV3020, Zeolyst Int., $\mathrm{Si} / \mathrm{Al}=16)$ to different 
treatments, namely: partial exchange of zeolite protons by monovalent $\left(\mathrm{Na}^{+}\right)$and divalent $\left(\mathrm{Co}^{2+}\right)$ cations at different exchange levels, and mild acid treatment $(1 \mathrm{M} \mathrm{HCl}$, $100^{\circ} \mathrm{C} / 12 \mathrm{~h}$ ). An exhaustive characterization by combining FTIR-pyridine and $\mathrm{NH}_{3}$-TPD techniques revealed the following changes in acidity depending on the treatment: a) the partial exchange with $\mathrm{Na}^{+}$preferentially neutralized the strongest Brønsted acid sites in the parent zeolite and produced weak Lewis acid sites unable to retain pyridine at a desorption temperature of $\left.250^{\circ} \mathrm{C}, \mathrm{b}\right)$ the exchange with $\mathrm{Co}^{2+}$ reduced the amount of Brønsted acid sites and created new Lewis acid sites of a moderate strength, and c) the acid treatment preferentially removed extraframework Al species (EFAL) leading to a drastic reduction in the density of EFAL-related strong Lewis acid sites with marginal changes in the amount and strength of the Brønsted acid sites. The set of prepared zeolites comprising samples with different amounts and strengths of Brønsted and Lewis acid sites were thus used to gain more insights into the role of zeolite acidity in both the dehydration of methanol and the syngas-to-DME (STD) reactions, in the later case using bifunctional catalysts comprising physical mixtures of a $\mathrm{CuO} / \mathrm{ZnO} / \mathrm{Al}_{2} \mathrm{O}_{3}$ methanol synthesis catalyst (CZA) and the zeolite.

Independent methanol dehydration experiments on the bare zeolites revealed that at the typical STD reaction temperature of $260^{\circ} \mathrm{C}$ the zeolite dehydration activity was mostly determined by the density of strong Brønsted acid sites (those able to retain pyridine at $400^{\circ} \mathrm{C}$ ) with a non-negligible contribution of EFAL-related strong Lewis acid sites (if present in the zeolite). The same conclusion hold true for the STD process when the overall STD reaction becomes controlled by the methanol dehydration step, that is, when using CZA/ZSM-5 hybrid catalysts with a low zeolite concentration (CZA:zeolite mass ratio of 10:1). In that case, a good linear relationship between the initial methanol dehydration rates obtained in the independent dehydration experiments 
on the bare zeolites and the CO conversion and/or DME yield during the STD runs was found.

Interestingly, divergent conclusions regarding the impact of zeolite acidity on the STD process were found depending on the specific methodology used for preparing the hybrids when the overall STD reaction is controlled by the methanol synthesis rate, that is, when using hybrids with a CZA:zeolite mass ratio of 2:1 and hence with an "excess" of acid sites. Under these conditions, no influence of zeolite acidity on the STD performance was observed for the hybrids prepared as simple physical mixtures of the pre-pelletized components. In that case, all the hybrids displayed similar and very high CO conversion and DME yield (ca. $90 \%$ and $58 \%$, respectively) with no signs of deactivation during a period of at least $50 \mathrm{~h}$ on stream. Contrarily, a gradual decline in CO conversion (and DME yield) with TOS was observed for equivalent hybrids prepared by grinding the CZA and zeolite powders prior to pelletizing, the rate of deactivation being largely affected by the characteristics of the particular ZSM-5 zeolite used. Thus, the most stable CZA/ZSM-5 hybrid prepared by the grinding method was that comprising the acid-treated EFAL-free zeolite, while the Na- and Co-exchanged zeolites lead to the hybrids with the lowest stability. Based on previous observations [38], the conflicting behavior of the hybrids prepared by different methods was tentatively attributed to the occurrence of detrimental interactions between the CZA and zeolite components during the grinding process that decreased the STD efficiency of the corresponding hybrids. Indeed, no such detrimental interactions could be expected for the most efficient hybrids prepared as mixtures of the pre-pelletized solids. Further work is in progress in order to elucidate the nature of such detrimental interactions and how they could be affected by the presence of EFAL and exchange cations in the zeolite dehydration component. 
The results of the present study clearly highlight the importance of the method of preparation of the hybrid catalysts and the regime of the STD process (control by the methanol synthesis or by the methanol dehydration steps) when trying to correlate the properties of the zeolite dehydration component (i.e. acidity) with the STD performance.

\section{Acknowledgments}

Financial support by the Comisión Interministerial de Ciencia y Tecnología (CICYT) of Spain through the Project CTQ2010-17988/PPQ is gratefully acknowledged. A. GarcíaTrenco thanks the Ministerio de Ciencia e Innovación (MICINN) of Spain for a predoctoral (FPI) scholarship.

\section{REFERENCES}

[1] T.A. Semelsberger, R.L. Borup, H.L Greene, J. Power Sources 156 (2006) 497-511.

[2] C. Arcoumanis, C. Bae, R. Crookes, E. Kinoshita, Fuel 87 (2008) 1014-1030.

[3] J.J. Spivey, Chem. Eng. Comm. 110 (1991) 123-142.

[4] Y. Lv, T. Wang, Ch. Wu, L. Ma, Y. Zhou, Biotech. Adv. 27 (2009) 551-554.

[5] J. Topp-Jorgense, US Patent 4,536,485 (1985), to Haldor Topsoe A/S Denmark.

[6] J.B. Hansen, F.H. Joensen, H.F.A. Topsoe, US Patent 5,189,203 (1993) to Haldor Topsoe A/S Denmark.

[7] J.L. Li, X.G. Zhang, T. Inui, Appl. Catal. A 147 (1996) 23-33.

[8] F. Yaripour, F. Baghaei, I. Schmidt, J. Perregaard, Catal. Commun. 6 (2005) 147152.

[9] T. Takeguchi, K. Yanagisawa, T. Inui, M. Inoue, Appl. Catal. 192 (2000) 201-209. 
[10] D. Mao, W. Yang, J. Xia, B. Zhang, G. Lu, J. Mol. Catal. A 250 (2006) 138-144.

[11] G.R. Moradi, S. Nosrati, F. Yaripor, Catal. Commun. 8 (2007) 598-606.

[12] Q. Ge, Y. Huang, F. Qiu, S. Li, Appl. Catal. A 167 (1998) 23-30.

[13] J.-H. Kim, M.J. Park, S.J. Kim, O.-S. Joo, K.-D. Jung, Appl. Catal. A 264 (2004) $37-41$.

[14] V. Vishwanathan, K.-W. Jun, J.-W. Kim, H.-S. Roh, Appl. Catal. A 276 (2004) $251-255$.

[15] J. Xia, D. Mao, B. Zhang, Q. Chen, Y. Tang, Catal. Lett. 98 (2004) 235-240.

[16] J. Ereña, R. Garoña, J.M. Arandes, A.T. Aguayo, J. Bilbao, Catal. Today 107-108 (2005) 467-473.

[17] L. Wang, Y. Qi, Y. Wei, D. Fang, S. Meng, Z. Liu, Catal. Lett. 106 (2006) 61-66.

[18] D. Mao, W. Yang, J. Xia, B. Zhang, Q. Song, Q. Chen, J. Catal. 230 (2005) 140149.

[19] M. Xu, J.H. Lunsford, D.W. Goodman, A. Bhattacharyya, Appl. Catal. A 149 (1997) 289-301.

[20] F.S. Ramos, A.M. Duarte de Farias, L.E.P. Borges, J.L. Monteiro, M.A. Fraga, E.F. Sousa-Aguiar, L.G. Appel, Catal. Today 101 (2005) 39-44.

[21] J. Xia, D. Mao, W. Tao, Q. Chen, Y. Zhang, Y. Tang, Microp. Mesop. Mater. 91 (2006) 33-39.

[22] D. Mao, J. Xia, Q. Chen, G. Lu, Catal. Commun. 10 (2009) 620-624.

[23] É. Sarkadi-Priboczki, N. Kumar, T. Salmi, Z. Kovács, D.Yu. Murzin, Catal. Lett. 93 (2004) 101-107.

[24] M.B. Sayed, R.A. Kydd, R.P. Cooney, J. Catal. 88 (1984) 137-149.

[25] K.S. Yoo, J.-H. Kim, M.-J. Park, S.-J. Kim, O.-S. Joo, K.-D. Jung, Appl. Catal. A 330 (2007) 57-62. 
[26] C. Baltes, S. Vukojević, F. Schüth, J. Catal. 258 (2008) 334-344.

[27] W. Song, R.E. Justice, C.A. Jones, V.H. Grassian, S.C. Larsen, Langmuir 20 (2004) 4696-4702.

[28] M.T. Twigg, M.S. Spencer, Top. Catal. 22 (2003) 191-203.

[29] K. Tohji, Y. Udagawa, T. Mizushima, A. Ueno, J. Phys. Chem. 89 (1985) 56715676.

[30] C.A. Emeis, J. Catal. 141 (1993) 347-354.

[31] L.B. Pierella, C. Saux, S.C. Caglieri, H.R. Bertorello, P.G. Bercoff, Appl. Catal. A 347 (2008) 55-61.

[32] J.M. Stencel, V.U.S. Rao, J.R. Diehl, K.H. Rhee, A.G. Dhere, J. Catal. 84 (1983) 109-118.

[33] M. Stöcker, Microp. Mesop. Mater. 29 (1999) 3-48.

[34] L. Kubelková, J. Nováková, K. Nedomová, J. Catal. 124 (1990) 441-450.

[35] G.R. Moradi, M. Nazari, F. Yaripur, Fuel Process. Technol. 89 (2008) 1287-1296.

[36] Y. Luan, H. Xu, C. Yu, W. Li, S. Hou, Catal. Lett. 115 (2007) 23-26.

[37] F.S.R. Barbosa, V.S.O. Ruiz, J.L.F. Monteiro, R.R. de Avillez, L.E.P. Borges, L.G. Appel, Catal. Lett. 126 (2008) 173-178.

[38] A. García-Trenco, A. Vidal-Moya, A. Martínez, Catal. Today (2011), doi:10.1016/j.cattod.2011.06.034.

[39] F.N. Lin, F. Penella, in Catalytic Conversion of Synthesis Gas to Alcohols and Chemicals (R.G. Herman, Ed.), Plenum Press, New York, 1984, p. 53. 
Table 1. Chemical composition (ICP-OES), relative crystallinity (XRD) and textural properties $\left(\mathrm{N}_{2}\right.$ physisorption) of the ZSM-5 samples.

\begin{tabular}{|c|c|c|c|c|c|}
\hline \multirow[b]{2}{*}{ Sample } & \multicolumn{2}{|c|}{ ICP-OES } & \multirow{2}{*}{$\begin{array}{c}\text { XRD } \\
\text { Crystallinity } \\
(\%)\end{array}$} & \multicolumn{2}{|c|}{$\mathrm{N}_{2}$ physisorption } \\
\hline & $\begin{array}{l}\mathrm{Si} / \mathrm{Al} \\
\text { ratio }\end{array}$ & $\begin{array}{l}\mathrm{M} / \mathrm{Al} \\
\text { ratio }\end{array}$ & & $\begin{array}{c}\text { BET area } \\
\left(\mathrm{m}^{2} / \mathrm{g}\right)\end{array}$ & $\begin{array}{l}V_{\text {micropore }} \\
\left(\mathrm{cm}^{3} / \mathrm{g}\right)\end{array}$ \\
\hline S1 & $\infty$ & - & 114 & 386 & 0.172 \\
\hline $\mathrm{HZ}$ & 16 & - & 100 & 396 & 0.162 \\
\hline $\mathrm{Na} 14 \mathrm{HZ}$ & 16 & 0.14 & - & 358 & 0.147 \\
\hline $\mathrm{Na} 27 \mathrm{HZ}$ & 16 & 0.27 & - & 350 & 0.144 \\
\hline $\mathrm{Na} 39 \mathrm{HZ}$ & 16 & 0.39 & 94 & 336 & 0.137 \\
\hline $\mathrm{Co} 8 \mathrm{HZ}$ & 16 & 0.08 & - & 377 & 0.153 \\
\hline Co15HZ & 16 & 0.15 & 91 & 367 & 0.151 \\
\hline $\mathrm{Co} 20 \mathrm{HZ}$ & 16 & 0.20 & 87 & 356 & 0.146 \\
\hline HZA & 18 & - & 99 & 385 & 0.153 \\
\hline
\end{tabular}


Table 2. Acidity of ZSM-5 samples as determined by FTIR of adsorbed pyridine.

\begin{tabular}{|c|c|c|c|c|c|c|}
\hline \multirow[b]{2}{*}{ Sample } & \multicolumn{6}{|c|}{ Amount of acid sites $(\mu \mathrm{mol} / \mathrm{g})$} \\
\hline & $\mathrm{B} 250^{\mathrm{a}}$ & $\mathrm{B} 350^{\mathrm{a}}$ & $\mathrm{B} 400^{\mathrm{a}}$ & $\mathrm{L} 250^{\mathrm{a}}$ & ${\mathrm{L} 350^{\mathrm{a}}}$ & $\mathrm{L}_{400}{ }^{\mathrm{a}}$ \\
\hline $\mathrm{HZ}$ & 255 & 197 & 160 & 56 & 47 & 45 \\
\hline $\mathrm{Na} 14 \mathrm{HZ}$ & 192 & 119 & 91 & 28 & 27 & 27 \\
\hline $\mathrm{Na} 27 \mathrm{HZ}$ & 129 & 92 & 68 & 18 & 18 & 17 \\
\hline $\mathrm{Na39HZ}$ & 81 & 65 & 38 & 22 & 17 & 16 \\
\hline Co8HZ & 184 & 151 & 108 & 80 & 53 & 42 \\
\hline Co15HZ & 122 & 113 & 75 & 92 & 84 & 65 \\
\hline $\mathrm{Co} 20 \mathrm{HZ}$ & 94 & 72 & 48 & 129 & 86 & 63 \\
\hline HZA & 252 & 207 & 172 & 8 & 8 & 7 \\
\hline
\end{tabular}




\section{Figure Captions}

Fig. 1. ${ }^{27}$ Al MAS NMR spectra for the parent and selected modified ZSM-5 zeolites. For a clearer comparison, all the spectra have been rescaled so as to show equivalent intensities for the $\mathrm{Al}^{\mathrm{IV}}$ signal at ca. $53 \mathrm{ppm}$.

Fig. 2. FTIR spectra in the pyridine region at a desorption temperature of $250^{\circ} \mathrm{C}$ for the parent and modified ZSM-5 samples.

Fig. 3. Correlations between the acid properties (as derived from FTIR-pyridine) and the atomic $\mathrm{M} / \mathrm{Al}$ ratio $(\mathrm{M}=\mathrm{Na}, \mathrm{Co})$ for partially exchanged ZSM-5 samples: a) total amount of Brønsted acid sites (B250), b) strong-to-total Brønsted acid sites ratio (B400/B250), c) total amount of Lewis acid sites (L250), and d) strong-to-total Lewis acid sites ratio (L400/L250).

Fig. 4. $\mathrm{NH}_{3}$-TPD profiles for the parent and modified ZSM-5 samples.

Fig. 5. Correlation between the initial methanol dehydration rate for the studied zeolites and the density of strong Brønsted acid sites as determined by FTIR-pyridine at a pyridine desorption temperature of $400^{\circ} \mathrm{C}$. Methanol dehydration conditions: $260^{\circ} \mathrm{C}, 1$ bar, $15-30 \mathrm{mg}$ of zeolite, $\mathrm{MeOH}: \mathrm{N}_{2}$ molar ratio= 20:80, total flow rate= $150 \mathrm{~mL} / \mathrm{min}$.

Fig. 6. Correlation between the CO conversion and DME yield obtained during STD experiments under methanol dehydration-controlled conditions for hybrids prepared by mixture of the pre-pelletized components in a CZA:zeolite mass ratio of 10:1 and the initial methanol dehydration rate on the bare zeolites. STD conditions: $260^{\circ} \mathrm{C}, 4.0 \mathrm{MPa}$, $\mathrm{GHSV}=1700 \mathrm{~mL}_{\text {syngas }} /\left(\mathrm{g}_{\text {cat }} \cdot \mathrm{h}\right)$, feed composition: 90 vol $\%$ syngas $\left(66 \% \mathrm{H}_{2} / 30 \% \mathrm{CO} / 4 \%\right.$ $\mathrm{CO}_{2}$ ) and 10 vol\% $\mathrm{Ar}$ (internal standard for $\mathrm{GC}$ analyses). Methanol dehydration conditions as given in the caption to Fig. 5. 
Fig. 7. CO conversion as a function of time-on-stream (TOS) in STD experiments performed under methanol synthesis-controlled conditions using CZA/ZSM-5 hybrids with a CZA:zeolite mass ratio of 2:1 prepared by mixing the pre-pelletized solids (a) and by grinding the individual components powders followed by pelletizing (b). STD reaction conditions as given in the caption to Fig. 6. The equilibrium $\mathrm{CO}$ conversion at the applied conditions has been calculated from the Gibbs free energy of the compounds using the Aspen Hysys 3.2 software and considering the main four reactions involved in STD.

Fig. 8. Selectivity to the main reaction products as a function of TOS in methanol synthesis-controlled STD experiments on selected hybrids with CZA:zeolite mass ratio of 2:1 prepared by the grinding method: a) $\mathrm{CZA} / \mathrm{Co} 20 \mathrm{HZ}(2: 1) \mathrm{G}$, and b) CZA/Na39HZ(2:1)G. STD conditions as in Fig. 6. 
Fig. 1

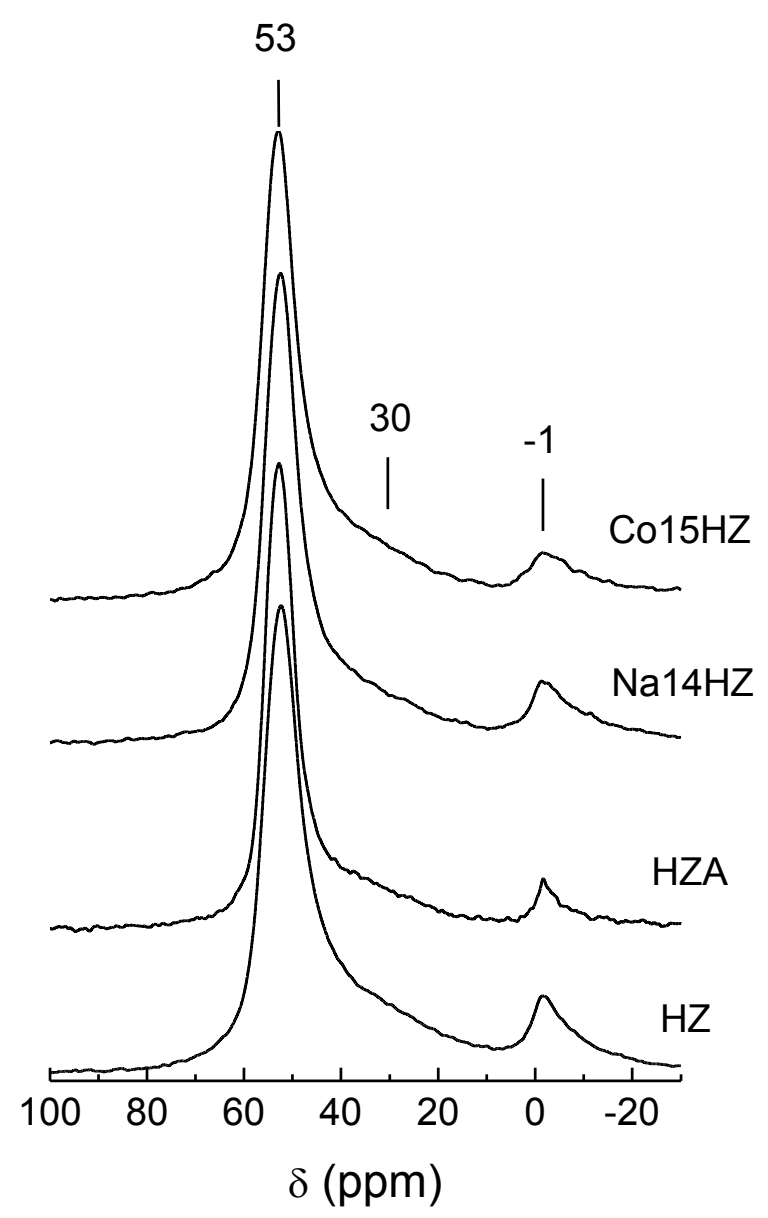


Fig. 2

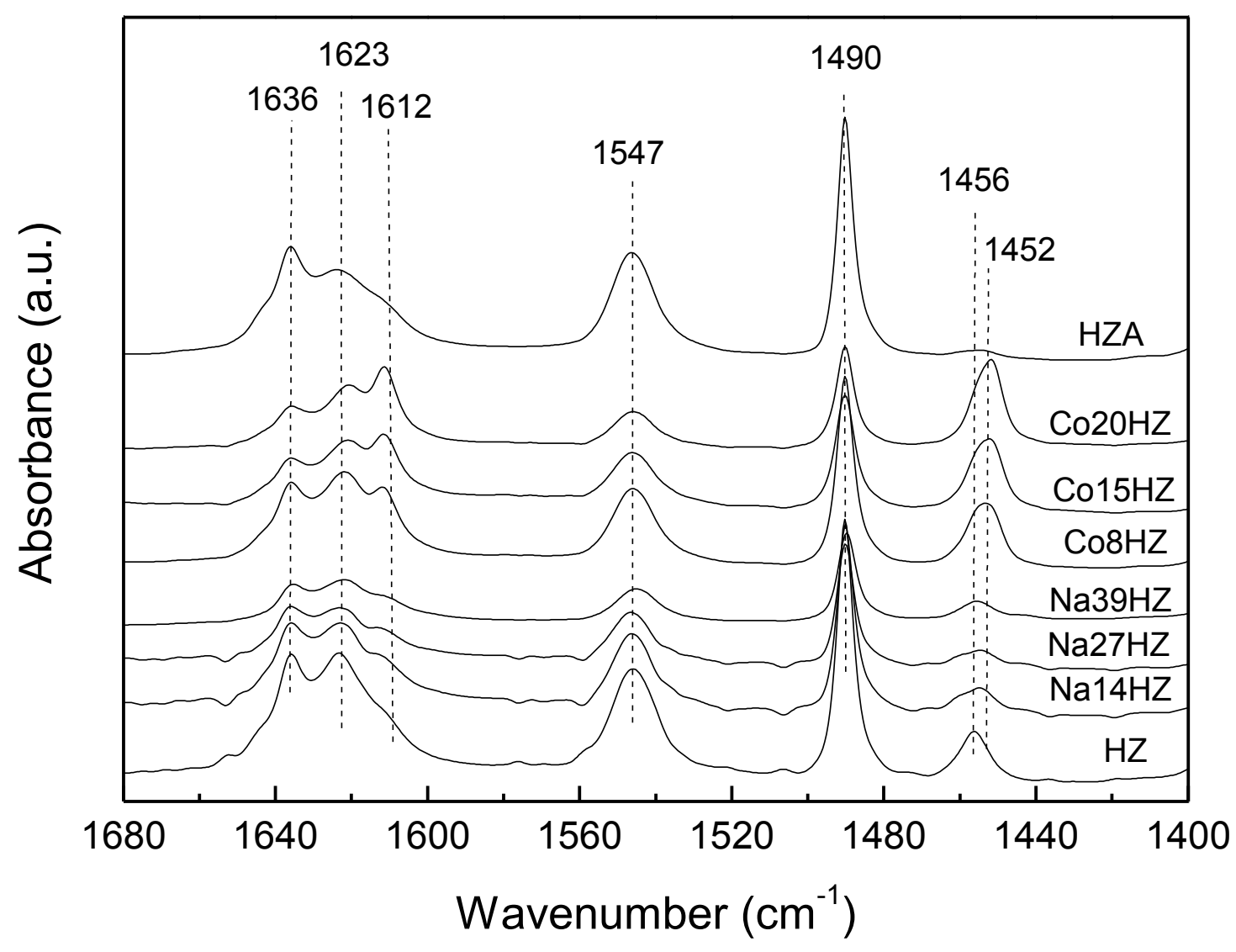


Fig. 3

(a)

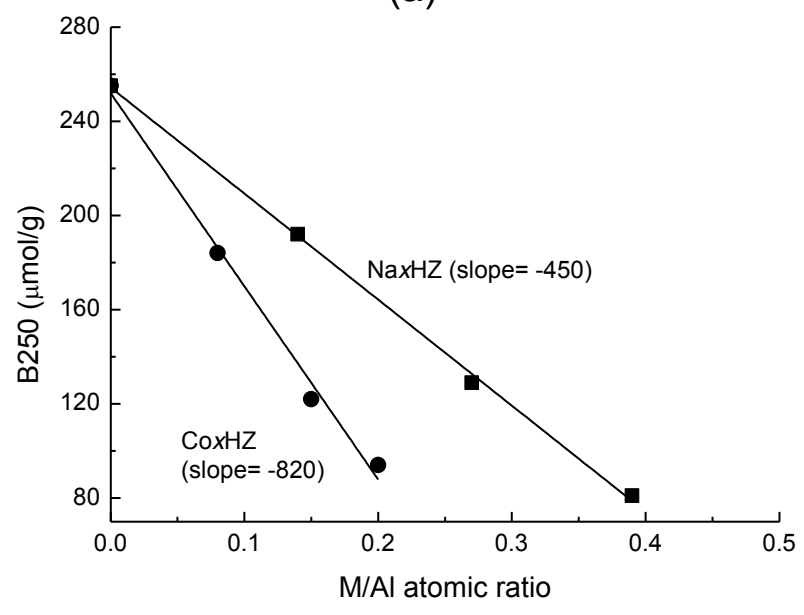

(c)

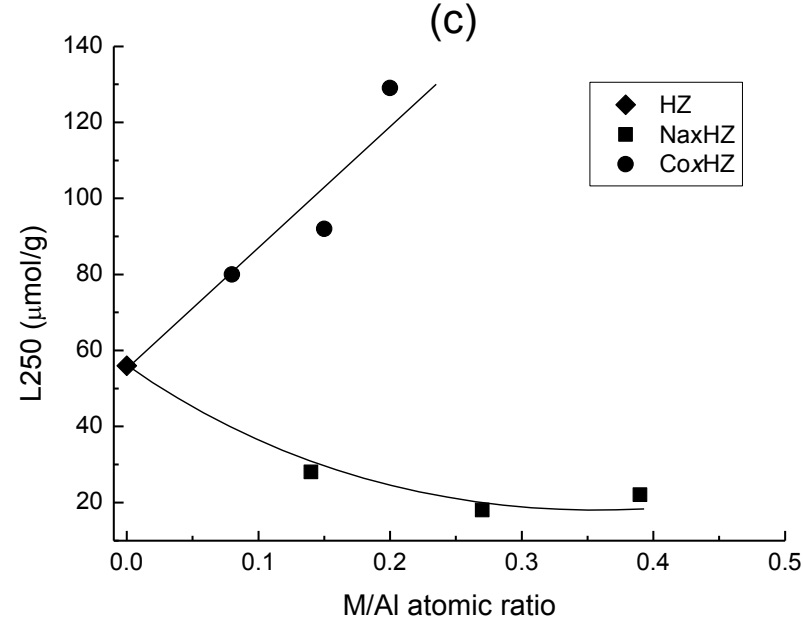

(b)

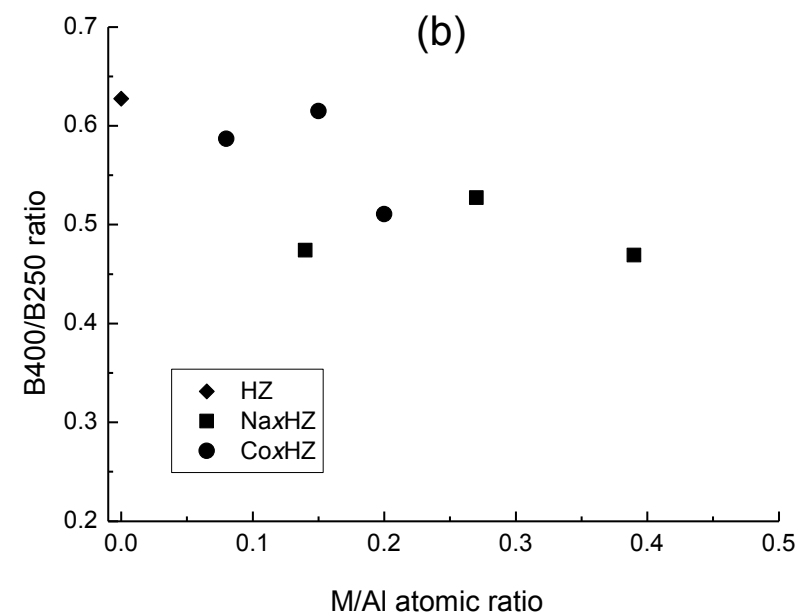

(d)

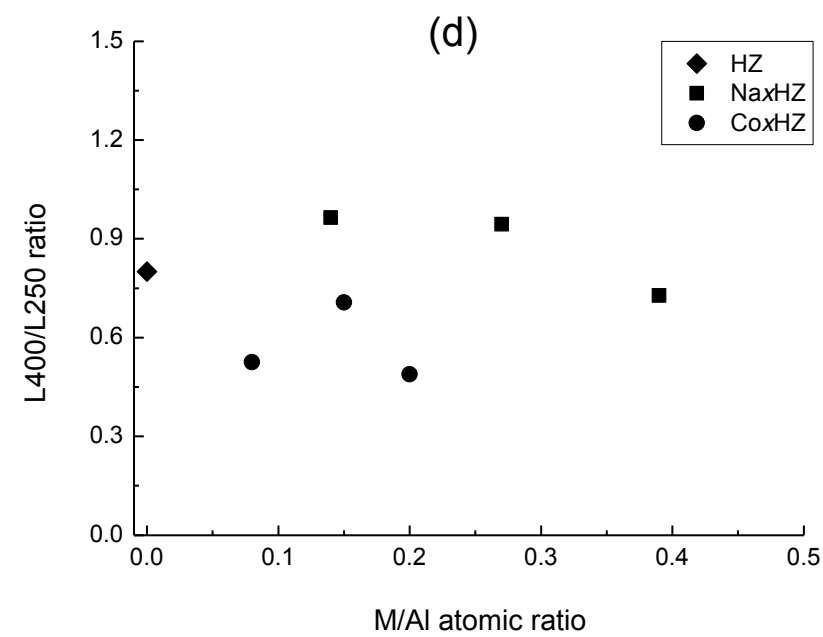


Fig. 4

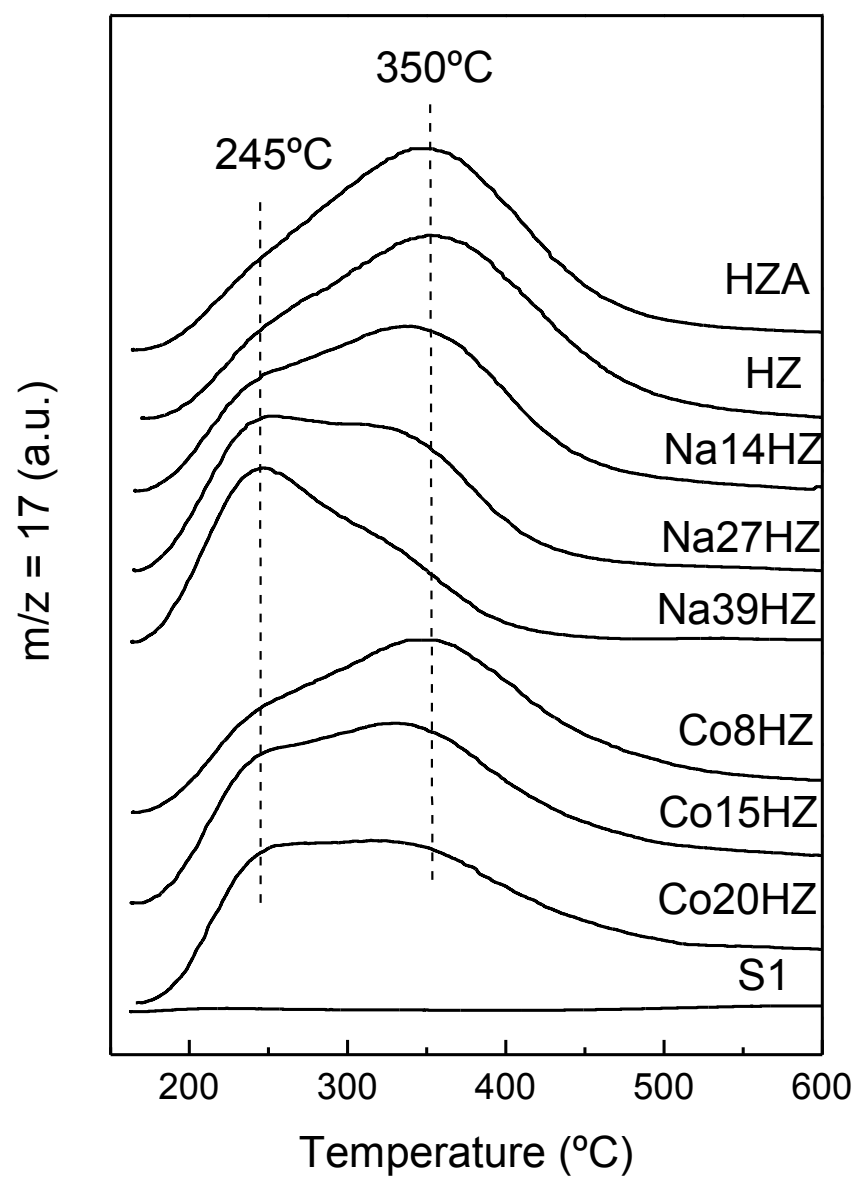


Fig. 5

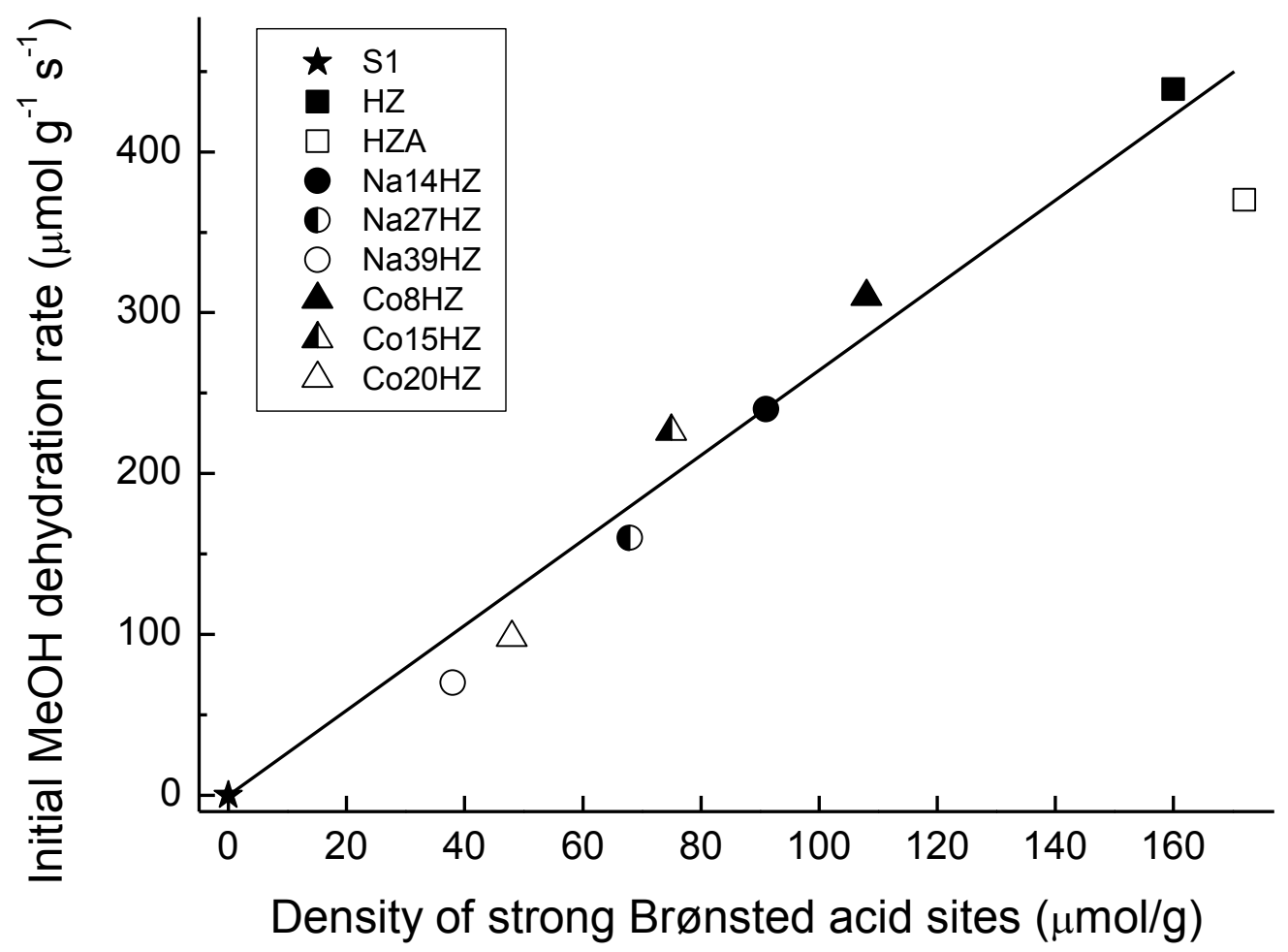


Fig. 6

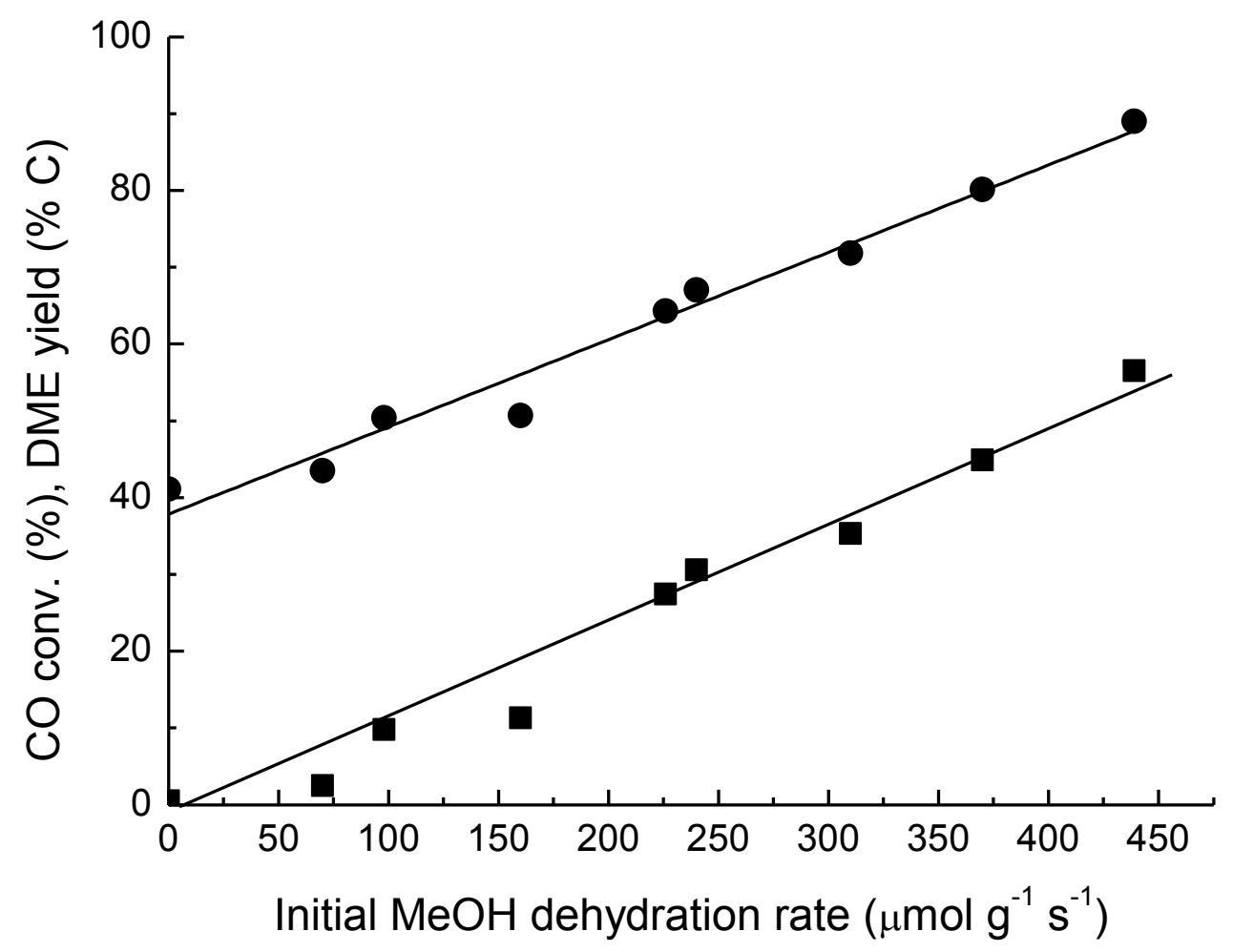


Fig. 7

a)

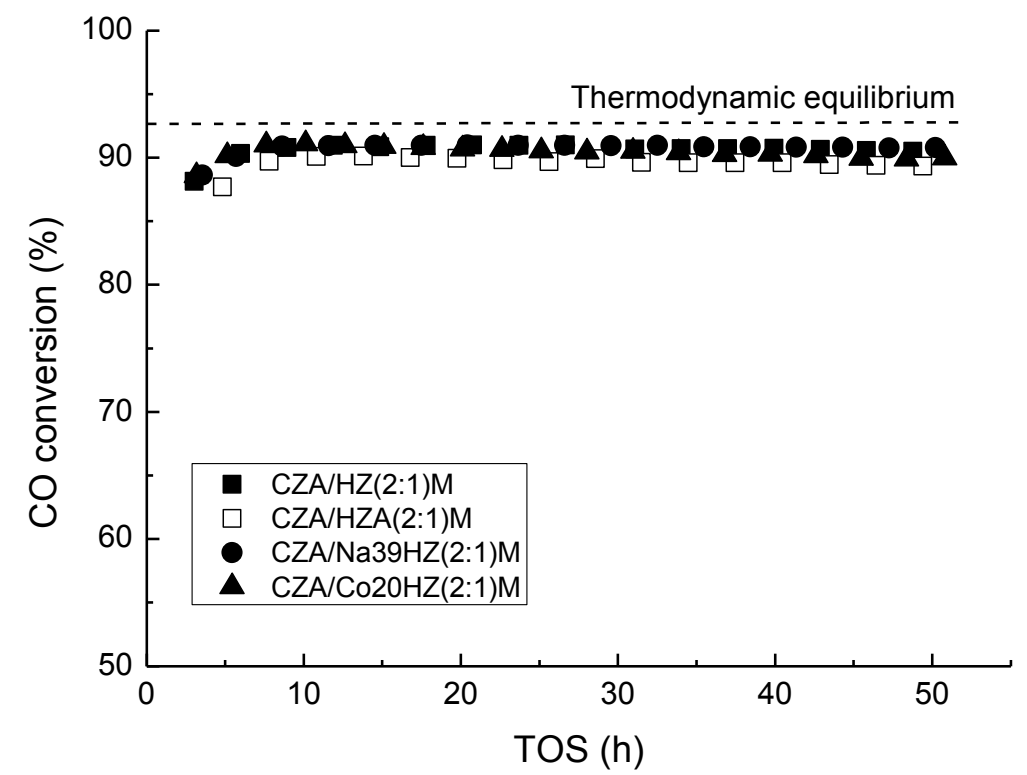

b)

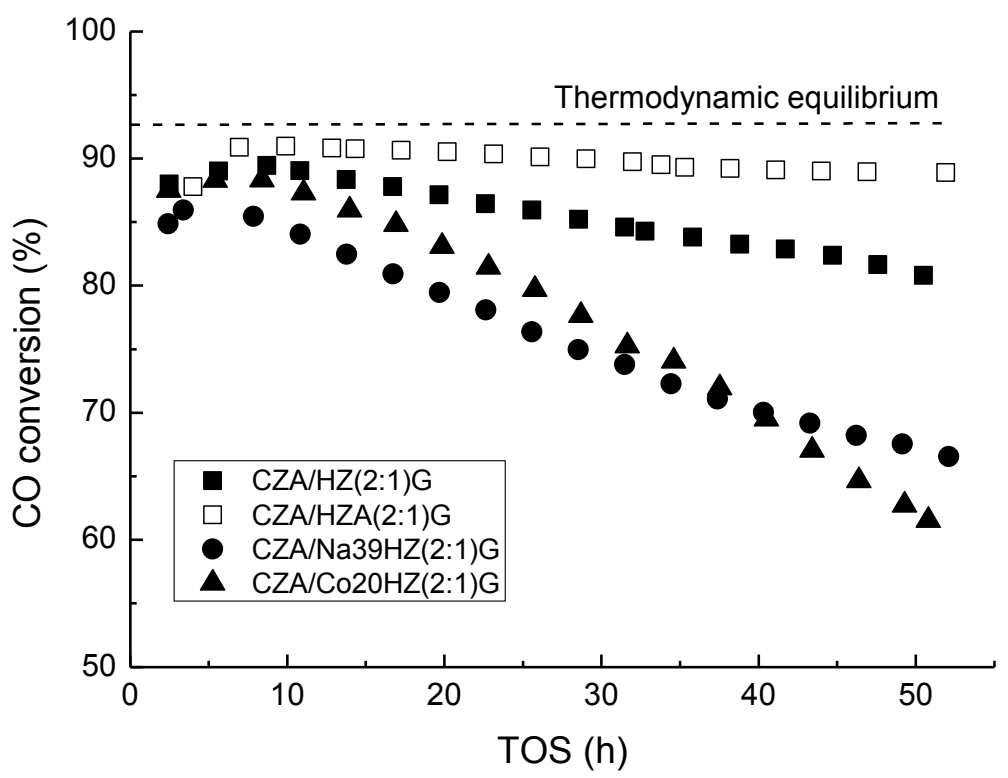


Fig. 8

a)

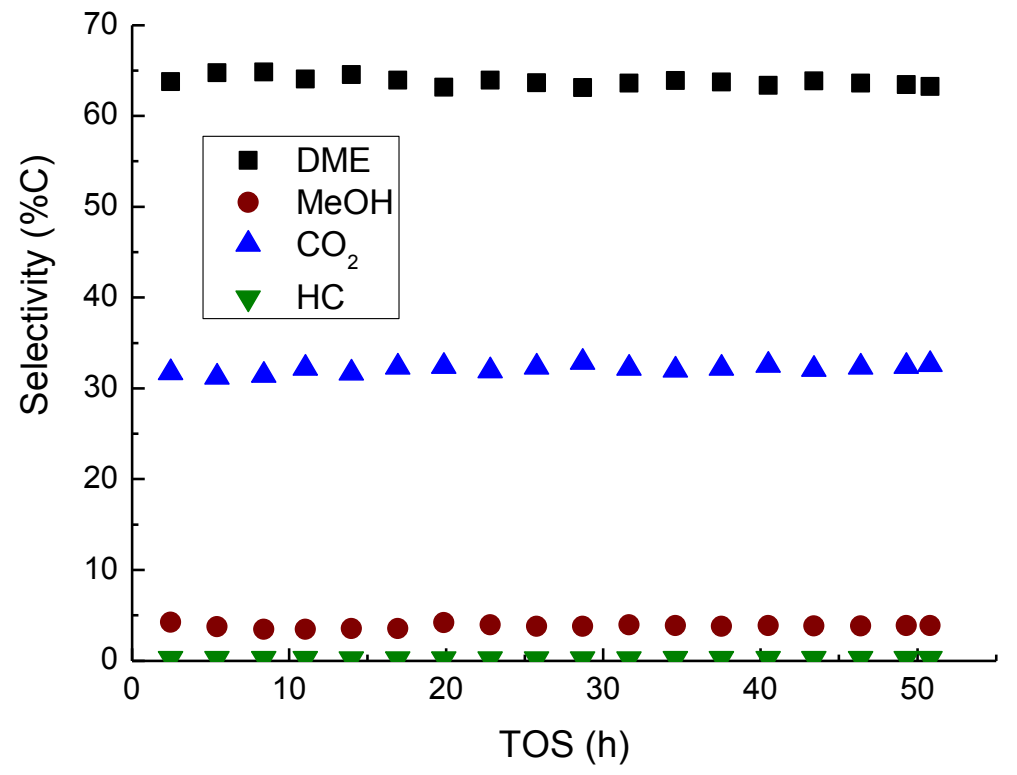

b)

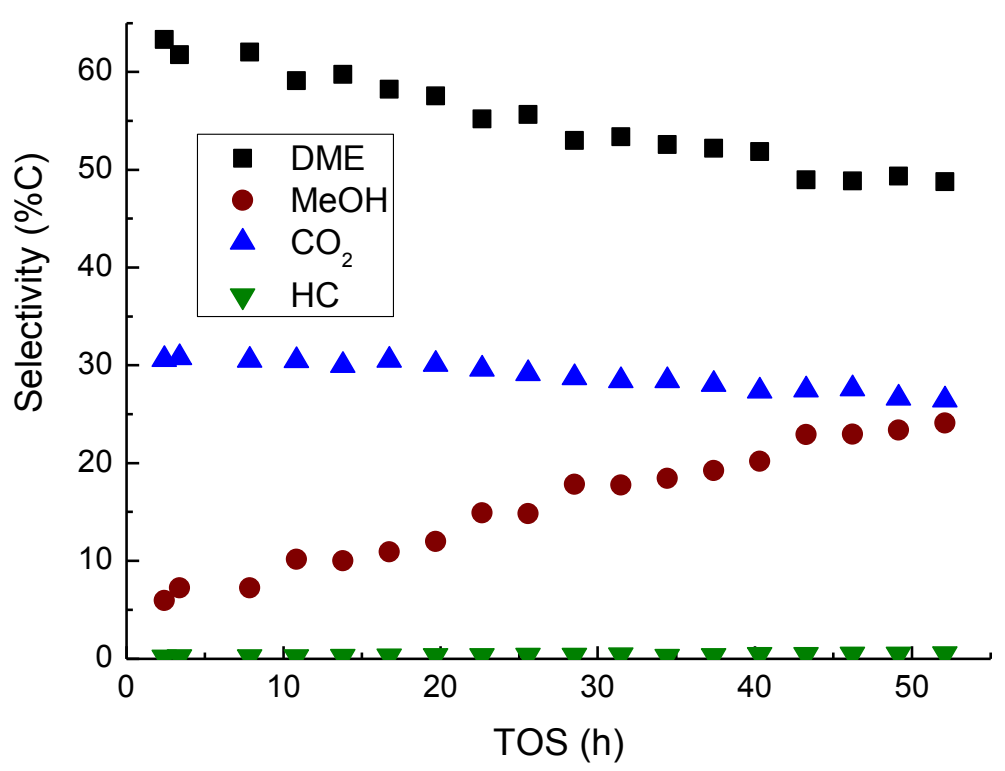




\section{Graphical Abstract}

\section{Syngas-to-DME (STD)}

Hybrids: CuZnAl/ZSM-5 (2:1)

\section{Methanol dehydration}
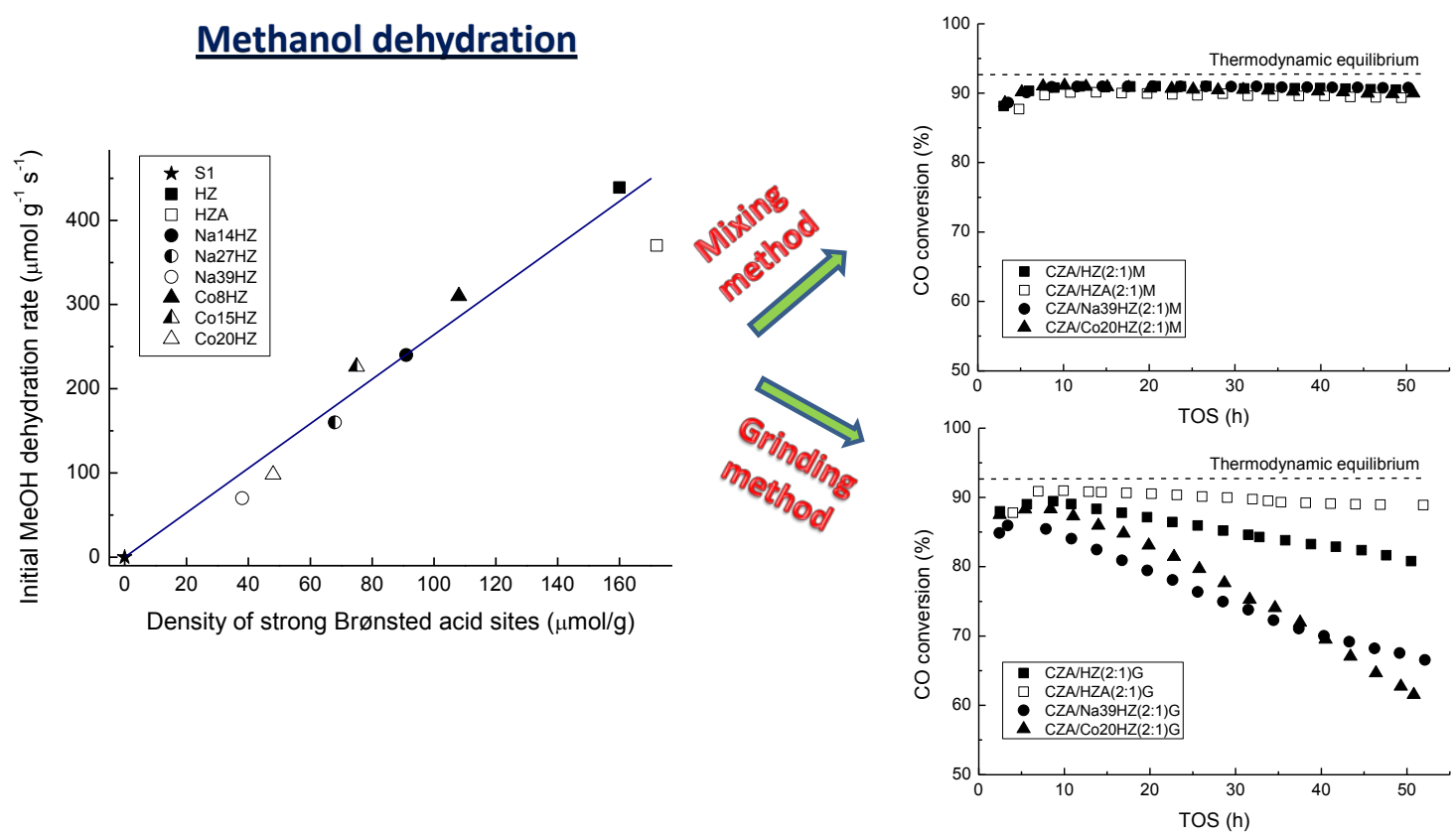


\section{Research highlights}

- The methanol dehydration activity of ZSM-5 at STD conditions is mostly driven by the density of strong Brønsted acid sites.

- EFAL-related strong Lewis acid sites do also contribute to the overall zeolite dehydration activity.

- The impact of zeolite acidity on STD performance largely depends on the specific method applied to prepare the CZA/zeolite hybrid catalysts.

- Mixing the pre-pelletized solids lead to the most efficient STD catalysts displaying high and stable $\mathrm{CO}$ conversion.

- Hybrids prepared by grinding the CZA and zeolite powders lead to poorer STD performance due to detrimental interactions between components. 
